\title{
INVARIANT RANDOM SUBGROUPS OVER NON-ARCHIMEDEAN LOCAL FIELDS
}

\author{
TSACHIK GELANDER AND ARIE LEVIT
}

\begin{abstract}
Let $G$ be a higher rank semisimple linear algebraic group over a non-Archimedean local field. The simplicial complexes corresponding to any sequence of pairwise non-conjugate irreducible lattices in $G$ are BenjaminiSchramm convergent to the Bruhat-Tits building. Convergence of the relative Plancherel measures and normalized Betti numbers follows. This extends the work $\mathrm{ABB}^{+} 17$ from real Lie groups to linear groups over arbitrary local fields. Along the way, various results concerning Invariant Random Subgroups and in particular a variant of the classical Borel density theorem are also extended.
\end{abstract}

\section{Contents}

1. Introduction

2. Semisimple linear groups over local fields

3. The Chabauty topology and Benjamini-Schramm convergence

4. On Zariski closure and the Chabauty topology

5. Borel density theorem for invariant random subgroups

6. Semisimple analytic groups are self-Chabauty-isolated

7. On Chabauty neighborhoods of lattices

8. Accumulation points of invariant random subgroups

9. Limit formula for normalized Betti numbers

10. Convergence of relative Plancherel measures

References

\section{INTRODUCTION}

We study lattices in semisimple analytic groups. The latter are roughly speaking direct products of simple linear groups over arbitrary local fields. Our goal is to establish certain asymptotic properties holding true for any sequence of pairwise non-conjugate irreducible lattices. The following statement is our main result.

Theorem 1.1. Let $G$ be a semisimple analytic group. Assume that $G$ is happy, has property $(T)$ and $\operatorname{rank}(G) \geq 2$. Let $\Gamma_{n}$ be a sequence of pairwise non-conjugate irreducible lattices in $G$. Then any accumulation point of the corresponding invariant random subgroups $\mu_{\Gamma_{n}}$ is equal to $\delta_{Z}$ for some central subgroup $Z$ in $G$.

A semisimple analytic group is happy if its non-Archimedean factor contains a topologically finitely generated compact open subgroup. This notion is motivated by BL04 and discussed in $\$ 2.3$ below. All semisimple analytic groups zero are 
happy in zero characteristic, and in positive characteristic all simply-connected semisimple analytic group are happy.

In positive characteristic the $\Gamma_{n}$ 's are required to be pairwise non-conjugate by automorphisms of $G$. Of course it is enough to assume that $\operatorname{Vol}\left(G / \Gamma_{n}\right) \rightarrow \infty$.

The Archimedean case of Theorem 1.1 has been established in $\left[\mathrm{ABB}^{+} 17\right.$ and our approach is basically the same as the one developed there. In particular the key strategy used in 88 towards our main theorem is similar to that of $\mathrm{ABB}^{+} 17$, §4.4]. However we are forced to deal with many issues that do not come up in the Archimedean case.

One of the difficulties encountered in the setting of arbitrary local fields has to do with positive characteristic phenomena, to which we pay particular attention. $p$-adic Lie groups are on the other hand easier to deal with than their real counterparts, and the mixed case usually requires little extra effort.

The property $(T)$ assumption in Theorem 1.1 is needed to rely on the deep theorem of Stuck and Zimmer. Let us note that, unlike the situation in $\mathrm{ABB}^{+} 17$, this is the only reason that property $(T)$ is essential for our approach. While it is used also in 8 when referring to GW97, this is done only to simplify the argument.

The property of accumulation points in question admits a geometric reformulation in terms of Benjamini-Schramm convergence. This and the notion of invariant random subgroups are explored in $\$ 3$ and used throughout this work.

1.1. Relative Plancherel measure convergence. One of the major achievements of $\mathrm{ABB}^{+} 17$ is a convergence formula for relative Plancherel measures with respect to a general uniformly discrete sequence of lattices. To be precise, let $\Gamma$ be a uniform lattice in $G$ so that the right quasi-regular representation $\rho_{\Gamma}$ of $G$ in $L^{2}\left(\Gamma \backslash G, \mu_{G}\right)$ decomposes as a direct sum of irreducible representations. Every irreducible unitary representation $\pi$ of $G$ appears in $\rho_{\Gamma}$ with finite multiplicity $m(\pi, \Gamma)$.

Definition 1.2. The normalized relative Plancherel measure of $G$ with respect to $\Gamma$ is an atomic measure on the unitary dual $\widehat{G}$ given by

$$
\nu_{\Gamma}=\frac{1}{\operatorname{vol}(\Gamma \backslash G)} \sum_{\pi \in \widehat{G}} m(\pi, \Gamma) \delta_{\pi} .
$$

It turns out that the following deep representation-theoretic statement follows from the geometric asymptotic property of the kind established in Theorem 1.1

Theorem 1.3. Let $G$ be a semisimple analytic group in zero characteristic. Fix a Haar measure on $G$ and let $\nu_{G}$ be the associated Plancherel measure on $\widehat{G}$. Let $\Gamma_{n}$ be a uniformly discrete sequence of lattices in $G$ with $\mu_{\Gamma_{n}}$ being weak-* convergent to $\delta_{\{e\}}$. Then

$$
\nu_{\Gamma_{n}}(E) \stackrel{n \rightarrow \infty}{\longrightarrow} \nu_{G}(E)
$$

for every relatively quasi-compact $\nu^{G}$-regular subset $E \subset \widehat{G}$.

The proof of Theorem 1.3 is essentially the same as $\left[\mathrm{ABB}^{+} 17,1.2,6.7\right]$. It relies on the Plancherel formula and the Sauvageot principle; see $\$ 10$. To the best of our knowledge, the latter principle appears in the literature only in zero characteristic, and for that reason we exclude positive characteristic local fields in Theorem 1.3.

Let $d_{\pi}$ denote the formal dimension of the irreducible unitary representation $\pi$ in the regular representation of $G$. 
Corollary 1.4. In the situation of Theorem 1.3 we have that moreover

$$
\frac{m\left(\pi, \Gamma_{n}\right)}{\operatorname{vol}\left(\Gamma_{n} \backslash G\right)} \stackrel{n \rightarrow \infty}{\longrightarrow} d_{\pi}
$$

for every $\pi \in \widehat{G}$.

Putting together the two Theorems 1.1 and 1.3 immediately gives the following.

Corollary 1.5. Let $G$ be a semisimple analytic group in zero characteristic. Assume that $G$ has property $(T)$ and that $\operatorname{rank}(G) \geq 2$. Then the conclusions of Theorem 1.3 and Corollary 1.4 apply to any sequence of uniformly discrete, torsion free, pairwise non-conjugate irreducible lattices.

Remark 1.6. Recall that in the non-Archimedean zero characteristic case any sequence of lattices in uniformly discrete. If $G$ is center-free then there is no need to assume torsion-freeness.

1.2. Normalized Betti numbers. Another achievement of $\mathrm{ABB}^{+} 17$ is a convergence formula for normalized Betti numbers, derived as a consequence of the convergence of relative Plancherel measures. We present here a direct argument applicable in the more combinatorial non-Archimedean case, relying on a beautiful result of Elek and avoiding Theorem 1.3 . This holds true in positive characteristic as well.

Recall that $b_{d}^{(2)}(G)$ denote the $L^{2}$-Betti numbers of the group $G$, c.f. Pet13.

Theorem 1.7. Let $G$ be a non-Archimedean semisimple analytic group. Let $\Gamma_{n}$ be any sequence of torsion-free uniform lattices in $G$ with $\mu_{\Gamma_{n}}$ being weak-* convergent to $\delta_{\{e\}}$. Then

$$
\lim _{n \rightarrow \infty} \frac{b_{d}\left(\Gamma_{n}\right)}{\operatorname{vol}\left(G / \Gamma_{n}\right)}=b_{d}^{(2)}(G)
$$

for all $d \in \mathbb{N} \cup\{0\}$.

Theorem 1.7 has been established for uniformly discrete sequences of lattices in the general framework of (not necessarily algebraic) locally compact totally disconnected groups in a recent interesting work by Petersen, Sauer and Thom [PST16]. The approach of [PST16] applies, to some extent, also to non-uniformly discrete families of lattices, in which case the $L^{2}$-Betti number gives a lower bound on the normalized Betti numbers. This includes in particular families of non-uniform lattices.

The following result is an immediate corollary of Theorems 1.3 and 1.7

Corollary 1.8. Let $G$ be a happy non-Archimedean semisimple analytic group with $\operatorname{rank}(G) \geq 2$ and property $(T)$. Let $X$ be the Bruhat-Tits building of $G$. Let $\Gamma_{n}$ be a uniformly discret 11 sequence of pairwise non-isomorphic, torsion-free, irreducible lattices in $G$. Then

$$
\lim _{n \rightarrow \infty} \frac{b_{d}\left(\Gamma_{n}\right)}{\left|V\left(\Gamma_{n} \backslash X\right)\right|}=b_{d}^{(2)}(X)
$$

for all $d \in \mathbb{N} \cup\{0\}$.

\footnotetext{
${ }^{1}$ The uniform discreteness assumption is redundant in characteristic 0.
} 
1.3. Borel density theorem for invariant random subgroups. Recall that an invariant random subgroup of $G$ is a Borel probability measure on the Chabauty space of closed subgroups which is invariant under conjugation. This generalizes the classical notion of lattices, and is used in the proof of our main result.

We extend two well-known and classical results to invariant random subgroups. The first is the Borel density theorem, saying that a lattice $\Gamma$ in a semisimple analytic group $G$ is Zariski dense provided that $G$ has no compact factors. The other one concerns closed subgroups $H \leq G$ such that $G / H$ admits finite $G$-invariant measure. For example, if $G$ is simple then such $H$ must be discrete. In zero characteristic the second result follows immediately from the first, but it requires an additional argument in positive characteristic — see [Mar91, II.5] or the more recent Pin04].

Theorem 1.9 (Borel density theorem for IRS). Let $k$ be a local field and $G$ a happy semisimple analytic group over $k$. Assume that $G$ has no almost $k$-simple factors of type $B_{n}, C_{n}$ or $F_{4}$ if $\operatorname{char}(k)=2$ and of type $G_{2}$ if $\operatorname{char}(k)=3$.

Let $\mu$ be an ergodic invariant random subgroup of $G$. Then there is a pair of normal subgroups $N, M \triangleleft G$ so that

$$
N \leq H \leq M, \quad H / N \text { is discrete in } G / N \text { and } \quad \bar{H}^{\mathrm{Z}}=M
$$

for $\mu$-almost every closed subgroup $H$ in $G$. Here $\bar{H}^{\mathrm{Z}}$ is the Zariski closure of $H$.

A variant of Theorem 1.9 for simple real Lie groups first appeared in $\mathrm{ABB}^{+} 17$. We use related ideas coming from Fursenberg's proof of the classical Borel density theorem in Fur76]. We would like to mention that geometric and Zariski density of invariant random subgroups was recently considered in the work [DGLL15].

One major difficulty peculiar to positive characteristic is the lack of a useful correspondence between closed subgroups and Lie subalgebras. Moreover, the Adrepresentation of a simple linear group need no longer be irreducible. We overcome these issues relying on a celebrated criterion by Pink Pin98 as well as a certain linearization technique developed in 4 .

While we believe Theorem 1.9 to be of independent interest, it is not used towards proving other results in this work.

1.4. Lattices in the Chabauty space. Let $G$ be a semisimple analytic group and $\Gamma$ an irreducible lattice in $G$. Our approach towards Theorem 1.1 suggests that we study small neighborhoods of $\Gamma$ in the Chabauty space associated to $G$. This shares a common theme with a previous work of the authors GL16 where an analogue question was resolved for uniform lattices. In particular, the following theorem can be seen as an extension of GL16 to the higher rank non-uniform case.

Theorem 1.10 (Chabauty local rigidity). If $\operatorname{rank}(G) \geq 2$ then the irreducible lattice $\Gamma$ admits a Chabauty neighborhood consisting of conjugates of $\Gamma$ by automorphisms of $G$. If $G$ is defined over zero characteristic then these automorphisms are inner.

Theorem 1.10 is essentially a variant of local rigidity, expressed in terms of small deformations in the Chabauty topology rather than the representation space of $\Gamma$ in $G$ which more algebraic in nature.

\footnotetext{
${ }^{2}$ Uniform lattices in isometry groups of proper, geodesically complete CAT(0) spaces are considered in [GL16]. This setup suffices for the non-Archimedean zero characteristic case.
} 
The following immediate corollary is to be compared with a closely related statement for lattices in semisimple Lie groups with property $(T)$ established in $\left.\mathrm{ABB}^{+} 17,4.6\right]$. In particular it holds true for any sequence of irreducible lattices which are pairwise non-isomorphic or with covolume tending to infinity. Let us reiterate that our current approach in this matter is independent of property $(T)$.

Corollary 1.11. If $\operatorname{rank}(G) \geq 2$ and $\Gamma_{n}$ is a sequence of irreducible lattices in $G$ pairwise non-conjugate by automorphisms of $G$ then the sequence $\mu_{\Gamma_{n}}$ is discrete in the space of extremal points of IRS $(G)$. If $G$ is defined over zero characteristic then it suffices to assume that the $\Gamma_{n}$ are pairwise non-conjugate.

Chabauty local rigidity can be used to deduce Wang's finiteness theorem -

Theorem 1.12 (Wang's finiteness). Assume that $\operatorname{rank}(G) \geq 2$, and if $G$ is defined over positive characteristic then assume that $G$ is simply connected.

Then $G$ admits only finitely many $\operatorname{Aut}(G)$-conjugacy classes of irreducible lattices with co-volume bounded by any fixed $v>0$. In the zero characteristic case $G$ admits finitely many inner conjugacy classes as above.

Theorem 1.12 is an extension of [GL16, Theorem 1.5] relying on Chabauty local rigidity as in our Theorem 1.10 and therefore applying to non-uniform lattices as well. This formulation of Wang's finiteness follows from the well-known results of Borel and Prasad [BP89]. The present approach is however potentially easier.

We remark that Theorem 1.12 complements Corollary 1.11 in the sense that any sequence of irreducible lattices considered in the latter corollary must have co-volume tending to infinity.

\section{Semisimple LiNEAR GRoups OVER LOCAL FIELDS}

For the reader's convenience we present and summarize well-known material used in this work. We define and discuss semisimple analytic groups. Several key results regarding these groups are mentioned, in particular a deep result of Pink Pin98.

2.1. Local fields. An Archimedean local field is either $\mathbb{R}$ or $\mathbb{C}$.

A non-Archimedean local field is a finite extension either of $\mathbb{Q}_{p}$ or of the field $\mathbb{F}_{q}((t))$ of formal Laurent series over $\mathbb{F}_{q}$, where $\mathbb{F}_{q}$ is the finite field with $q$ elements and $q=p^{n}$ is a prime power. Note that $\operatorname{char}\left(\mathbb{Q}_{p}\right)=0$ and $\operatorname{char}\left(\mathbb{F}_{q}((t))\right)=p$. The prime field of $k$ is $\mathbb{Q}$ in the first case and $\mathbb{F}_{p}$ in the second case. Let $\mathcal{O}$ denote the valuation ring of $k$ and $\pi$ a uniformizer element. For example, if $k=\mathbb{Q}_{p}$ then $\mathcal{O}=\mathbb{Z}_{p}$ and one may take $\pi=p$, and if $k=\mathbb{F}_{q}((t)), \mathcal{O}=\mathbb{F}_{q}[[t]]$ and one may take $\pi=t$.

Remark 2.1. In a few arguments below our main efforts are intended to deal with the positive characteristic case, the zero characteristic case being easier.

2.2. Semisimple analytic groups. Our main objects of study are semisimple analytic groups.

Definition 2.2. Let $k$ be a local field and $\mathbb{G}$ a connected $k$-isotropic $k$-simple linear $k$-algebraic group.

- A simple analytic group is a group of the form $\mathbb{G}(k)$.

- A semisimple analytic group is an almost direct product of finitely many simple analytic groups, possibly over different local fields. 
Note that if $k$ is a local field and $\mathbb{G}$ is a connected semisimple linear $k$-algebraic group without $k$-anisotropic factors then $\mathbb{G}(k)$ is a semisimple analytic group. Such a group is indeed analytic in the sense of e.g. [Ser09.

A semisimple analytic group is respectively non-Archimedean/has zero characteristic/simply connected if all of its simple analytic almost direct factors are nonArchimedean/defined over a local field of zero characteristic/are simply connected.

Associated to a semisimple analytic group $G$ are its universal covering group $\widetilde{G}$ and adjoint group $\bar{G}$. There are central $k$-isogenies $\widetilde{G} \stackrel{\widetilde{p}}{\rightarrow} G \stackrel{\bar{p}}{\rightarrow} \bar{G}$ and this data is unique up to a $k$-isomorphism [Mar91, I.4.11].

2.3. The subgroup $G^{+}$and happy semisimple analytic groups. Let $G$ be a semisimple analytic group. Denote by $G^{+}$the subgroup of $G$ generated by its unipotent elements. This notion is rather important for our needs, especially when $G$ is not simply connected. We summarize some of the properties of $G^{+}$and refer to Mar91, I.1.5,I.2.3] and the references therein for more details.

If $G$ is simply connected then $G=G^{+}$. If $G$ is Archimedean then $G^{+}$is the connected component $G_{0}$ at the identity. In general $G / G^{+}$is a compact abelian group. The group $G^{+}$admits no proper finite index subgroups.

The group $G^{+}$is useful in the description of normal subgroups -

Proposition 2.3. Let $N$ be a subgroup of $G$ normalized by $G^{+}$. Then $N$ is normal in $G$ and there is an almost direct factor $M$ of $G$ such that $M^{+} \leq N$ and $N M / M$ is central in $G / M$.

Proof. Let $H$ be any almost $k$-simple factor of $G$. The projection of $N$ to $H$ as well as the intersection of $N$ with $H$ are normalized by $H^{+}$. Therefore these two subgroups of $H$ are either central or contain $H^{+}$[Mar91, I.1.5.6]. The proposition follows from these facts and Goursat's lemma in elementary group theory.

We introduce a certain notion of happiness that implies that the subgroup $G^{+}$is particularly nicely behaved and will be useful in dealing with non-simply-connected semisimple analytic groups.

Definition 2.4. A simple analytic group $G$ is happy if $\operatorname{char}(k)$ does not the divide $|Z|$ where $Z$ is the kernel of the map $\widetilde{G} \rightarrow G$. A semisimple analytic group is happy if all of its almost direct factors are.

Note that a simply connected or a zero characteristic semisimple analytic group is automatically happy.

Theorem 2.5 (Barnea-Larsen). The following are equivalent for a semisimple analytic group $G$.

(1) $G$ is happy,

(2) The central k-isogeny $\widetilde{p}: \widetilde{G} \rightarrow G$ is separable, or equivalently is an open map in the Hausdorff topology,

(3) $G / G^{+}$is a finite abelian group,

(4) Some (equivalently every) compact open subgroup in the non-Archimedean factor of $G$ is finitely generated.

The work of Barnea and Larsen focuses on property (44). Some of the equivalences between (1), (2) and (3) are discussed already e.g. in [BT73. 
Proof. The Archimedean factor $G_{\mathrm{c}}$ of $G$ is happy by definition. The map $\widetilde{p}_{\mathrm{c}}$ : $\widetilde{G}_{\mathrm{c}} \rightarrow G_{\mathrm{c}}$ is a local diffeomorphism. The subgroup $G_{\mathrm{c}}^{+}$is equal to the connected component $G_{\mathrm{c}, 0}$ at the identity of $G_{\mathrm{c}}$ and $G_{\mathrm{c}} / G_{\mathrm{c}, 0}$ is finite. Therefore (11), (2) and (3) all hold automatically for the Archimedean factor.

Recall that $\widetilde{p}(\widetilde{G})=G^{+}$Mar91, I.1.5.5]. The equivalence of (11), (2) and (3) for the non-Archimedean factor is established in BL04, 4.2], and this is equivalent to (4) by [BL04, 4.5]. The fact that $G / G^{+}$is abelian is contained in Mar91, I.1.5].

We remark that [BL04] relies on Pink's theorem [Pin98] discussed below.

2.4. Pink's theorem. We discuss a deep result of Pink, which is in a sense a generalization to positive characteristic of certain results due to Weyl in the real case and Chevalley in the $p$-adic case on compact subgroups of algebraic groups.

Let $k$ be a local field 3 and $G$ a simple analytic group over $k$. The following notation will be useful in making a concise statement of Pink's results.

Definition 2.6. Let $\rho$ be a $k$-linear representation of $G$. For every subgroup $\Gamma$ of $G$ the trace field $\mathcal{T F}_{\rho}(\Gamma)$ is the closed subfield of $k$ generated by the traces $\operatorname{tr} \rho(g)$ for all $g \in \Gamma$.

We recall two notions used below. First, let $\mathcal{R}_{k / l}$ denote Weil's restriction of scalars functor from $k$ to its subfield $l$. Next, recall from [Pin98, §1] that the commutator on $G$ factors through a generalized commutator map [,] $: G \times G \rightarrow \widetilde{G}$.

Theorem 2.7 (Pink). Assume that $G$ is adjoint, absolutely simple and has no non-standard isogenie 5 .

Then $G$ admits a k-linear representation $\rho$ such that for every compact Zariski dense subgroup $\Gamma$ the field $k$ is a finite extension of the trace field $\mathcal{T} \mathcal{F}_{\rho}(\Gamma)$.

If the closure of $\widetilde{[\Gamma, \Gamma]}$ in $\widetilde{G}$ is moreover Zariski dense in $\mathcal{R}_{k / \mathcal{T} \mathcal{F}_{\rho}(\Gamma)}(\widetilde{G})$ then it is compact and open in $\widetilde{G}$.

The notation $H \otimes k$ below stands for $H$ regarded as a $k$-linear group.

Proof. The Main Theorem 0.2 of Pin98 applies to any compact Zariski dense subgroup $\Gamma$ of $G$. It provides a closed subfield $l \subset k$ such that $k / l$ is a finite extension, an $l$-linear adjoint absolutely simple group $H$ and a $k$-isogeny $\varphi: H \otimes k \rightarrow$ $G$. Since $G$ admits no non-standard isogenies the map $\varphi$ is in fact a $k$-isomorphism Pin98, 1.7]. Let $\widetilde{H}$ and $\widetilde{\varphi}$ denote the universal cover of $H$ and the corresponding $k$-isomorphism $\widetilde{\varphi}: \widetilde{H} \otimes k \rightarrow \widetilde{G}$, respectively. Moreover there is a compact open subgroup $O \subset \widetilde{H}(l)$ so that $\widetilde{\varphi}(O)$ is equal to the closure of $\widetilde{[\Gamma, \Gamma]}$ in $\widetilde{G}$.

The existence of a suitable $k$-representation $\rho$ of $G$ independent of $\Gamma$ and the fact that $l$ is equal to $\mathcal{T F}_{\rho}(\Gamma)$ follow from [Pin98, 0.6(a)]. We are using the fact that $\tilde{\varphi}$ is a $k$-isomorphism in our case [Pin98, p. 503].

Assume moreover that $\Gamma$ regarded as a subgroup of the group of $l$-points of $\mathcal{R}_{k / l}(\widetilde{G})$ is Zariski-dense. This implies that $l$ is in fact equal to $k$ [Pin04, 2.4]. Therefore $\widetilde{\varphi}(O)$ is a compact open subgroup of $\widetilde{G}$.

\footnotetext{
${ }^{3}$ The local field $k$ in Pink's theorem is not assumed to be non-Archimedean.

${ }^{4} G$ is absolutely simple if it is simple over the algebraic closure $k_{\text {alg }}$ of $k$.

${ }^{5} G$ has no non-standard isogenies unless $\operatorname{char}(p)=2$ and $G$ is of type $B_{n}, C_{n}$ or $F_{4}$ or $\operatorname{char}(p)=3$ and $G$ is of type $G_{2}$. See $[\overline{P i n 98}, \S 1]$ for more details.
} 
In particular, if the adjoint group $G$ is happy then the second conclusion of Theorem 2.7 implies that $\Gamma$ itself is open in $G$. We will not be using this observation.

Proposition 2.8. Assume that $G$ is adjoint and absolutely simple. Let $\Gamma$ be a closed subgroup and $V \subset U$ a pair of compact open subgroups in $G$. If $\Gamma \cap V$ is Zariski dense then $\mathcal{T F}_{\rho}(\Gamma \cap U)=\mathcal{T F}_{\rho}(\Gamma \cap V)$ where $\rho$ is as in Theorem 2.7.

Proof. This follows from the uniqueness established in [Pin98, 0.2.(b)].

Remark 2.9. One of the main difficulties in Pink's theorem is the possibility that the adjoint representation of $G$ might not be irreducible in positive characteristic. There is a simpler proof provided that Ad is irreducible, see [Pin98, 0.7].

The same problem arises when proving discreteness for invariant random subgroups in our Theorem 1.9, and this was part of the motivation in considering Zariski closure instead of Lie algebras in \$4 below.

2.5. Maximal compact open subgroups. If $G$ is a simply connected simple analytic group then a maximal compact open subgroup of $G$ is maximal. This result is called Tits' theorem [Pra82]. The following is true in the general semisimple case.

Theorem 2.10. Let $G$ be a semisimple analytic group and $U$ an open subgroup in $G$. Then there is a normal subgroup $N \triangleleft G$ so that $N \leq U$ and $U / N$ is compact.

Proof. Since $U$ is open the quotient $G / U$ is discrete. Therefore the discrete metric on $G / U$ is $G$-invariant. The result now follows directly from Theorem 6.1 of [BG14.

As an immediate corollary of Theorems 2.5 and 2.10 and Proposition 2.3 we obtain the following fact.

Corollary 2.11. Let $G$ be a happy semisimple analytic group and $K$ a maximal compact subgroup of $G^{+}$. Then there are only finitely many intermediate subgroups $H$ satisfying $K \leq H \leq G$.

2.6. Automorphisms of semisimple analytic groups. Let $G$ be a semisimple analytic group. The following facts regarding topological automorphisms of $G$ is well known. However, we are not aware of a specific reference in the literature.

Consider the group $\operatorname{Aut}(G)$ of the topological group automorphisms of $G$ with the Braconnier topology Bra45] and the group of outer automorphisms Out $(G)=$ $\operatorname{Aut}(G) / \operatorname{Inn}(G)$ with the quotient topology.

Proposition 2.12. The group of outer automorphisms $\operatorname{Out}(G)$ is compact.

Proof. We first show how to reduce the problem to the simply connected case. Recall that $\widetilde{G}$ is the universal covering group of $G$ and $p: \widetilde{G} \rightarrow G$ is the corresponding central $k$-isogeny with $\operatorname{ker} p$ being central in $\widetilde{G}$. Since the center $Z(\widetilde{G})$ is a characteristic subgroup, in effect $\operatorname{Aut}(\widetilde{G})$ is acting on the finite set $Z(\widetilde{G})$. Let $\operatorname{Aut}_{p}(\widetilde{G}) \operatorname{denote}$ the finite index closed subgroup of $\operatorname{Aut}(\widetilde{G})$ consisting of these automorphisms fixing $\operatorname{ker}(p)$ point-wise. Clearly $\operatorname{Inn}(\widetilde{G}) \leq \operatorname{Aut}_{p}(\widetilde{G}) \leq \operatorname{Aut}(\widetilde{G})$.

The map $p$ induces a continuous and surjective map $p_{*}: \operatorname{Aut}_{p}(\widetilde{G}) \rightarrow \operatorname{Aut}(G)$. Observe that

$$
\operatorname{Inn}(\widetilde{G}) \cong \widetilde{G} / Z(\widetilde{G}), \quad \operatorname{Inn}(G) \cong G / Z(G) \quad \text { and } \quad Z(G) \cong Z(\widetilde{G}) / \operatorname{ker}(p)
$$


and $p_{*}$ restricts to a bijection from $\operatorname{Inn}(\widetilde{G})$ to $\operatorname{Inn}(G)$. In particular $p_{*}$ induces a surjective continuous map $p_{*}: \operatorname{Out}_{p}(\widetilde{G}) \rightarrow \operatorname{Out}(G)$. Since $\operatorname{Out}_{p}(\widetilde{G})$ is closed in $\operatorname{Out}(\widetilde{G})$ it suffices to show that $\operatorname{Out}(\widetilde{G})$ is compact.

The simply connected group $\widetilde{G}$ is the direct product of its almost $k_{i}$-simple factors $\widetilde{G}_{1}, \ldots, \widetilde{G}_{n}$ defined over various local fields $k_{i}$ Mar91, I.4.10]. Out $(\widetilde{G})$ admits a finite index subgroup equal to $\prod_{i=1}^{n} \operatorname{Out}\left(\widetilde{G}_{i}\right)$ preserving each factor.

The theory of abstract homomorphisms of isotropic algebraic groups BT73, Mar91, I.1.8] shows that every automorphism of the topological group $\widetilde{G}_{i}$ is determined by an algebraic $k_{i}$-automorphism and an automorphism of the local field $k_{i}$. Recall that the group of $k_{i}$-automorphisms of $\widetilde{G_{i}}$ is the semi-direct product of the group of inner $k_{i}$-automorphisms by a finite group [Tit66, 1.5.6]. We conclude that $\operatorname{Out}(\widetilde{G})$ is compact.

Remark: In zero characteristic the group $\operatorname{Out}(G)$ of outer automorphisms is actually finite.

Corollary 2.13. Topological group automorphisms of $G$ preserve Haar measure.

Proof. The modular function $\triangle: \operatorname{Aut}(G) \rightarrow \mathbb{R}_{>0}^{*}$ is given by $\triangle(\alpha) \mu_{G}=\alpha_{*} \mu_{G}$ where $\mu_{G}$ is a fixed Haar measure on $G$. $\triangle$ is continuous with respect to the Braconnier topology [Bra45, IV.§3.]. Since $G$ is unimodular $\triangle(\operatorname{Inn}(G))=1$ and the result follows from Proposition 2.12

\section{The Chabauty topology and Benjamini-Schramm convergence}

In this section we study the Chabauty topology, invariant random subgroups, spaces of quotients and Benjamini-Schramm convergence and explore the relationship between these various notions. We focus on comparing three closely related properties of lattice sequences - being weakly trivial, IRS convergence to $\left.\delta_{\{} e\right\}$ and Benjamini-Schramm convergence of the quotients.

3.1. The Chabauty topology. Let $G$ be any second countable locally compact groun 6 .

Definition 3.1. The Chabauty space $\operatorname{Sub}(G)$ of all closed subgroups of $G$ is equipped with the Chabauty topology generated by the following sub-basis sets

- $\mathcal{O}_{1}(K)=\{H \leq G$ closed : $H \cap K=\emptyset\}$ for every compact subset $K \subset G$, - $\mathcal{O}_{2}(U)=\{H \leq G$ closed : $H \cap U \neq \emptyset\}$ for every open subset $U \subset G$.

The space $\operatorname{Sub}(G)$ is compact and admits a continuous $G$-action by conjugation.

Definition 3.2. An invariant random subgroup of $G$ is a Borel probability measure on $\operatorname{Sub}(G)$ which is invariant under conjugation by $G$.

Let IRS $(G)$ denote the space of all invariant random subgroups of $G$ equipped with the weak-* topology. By Riesz' representation theorem and Alaoglu's theorem, $\operatorname{IRS}(G)$ is a compact space.

\footnotetext{
${ }^{6}$ From now on we write l.c.s.c. for locally compact second countable.
} 
3.2. Weak triviality and weak uniform discreteness. Let $G$ be any l.c.s.c. group. We study weak-* convergence to $\delta_{\{e\}}$ in the space $\operatorname{IRS}(G)$ and relate this to the notion of weak uniform discreteness.

Definition 3.3. Given $\mu \in \operatorname{IRS}(G)$ and a subset $A \subset G$ denote

$$
p_{\mu}(A)=\operatorname{Prob}_{\mu}(\{H \leq G \text { closed }: A \cap H=\emptyset\})
$$

A sequence of invariant random subgroups $\mu_{n} \in \operatorname{IRS}(G)$ is called

- weakly trivial if $\lim _{n \rightarrow \infty} p_{\mu_{n}}(Q \backslash\{e\})=1$ for every compact subset $Q \subset G$, and

- weakly uniformly discrete if for every $\varepsilon>0$ there is an identity neighborhood $U_{\varepsilon} \subset G$ so that $p_{\mu_{n}}\left(U_{\varepsilon} \backslash\{e\}\right)>1-\varepsilon$ for all $n \in \mathbb{N}$.

The notion of weak uniform discreteness was introduced in Gel15] where it is used to provide a simple proof for a generalization of the Kazhdan-Margulis theorem. In fact all discrete invariant random subgroups in zero characteristic semisimple analytic groups are weakly uniformly discrete Gel15.

Proposition 3.4. A weakly trivial sequence of invariant random subgroups weak-* converges to $\delta_{\{e\}} \in \operatorname{IRS}(G)$. The converse direction holds provided that the sequence is weakly uniformly discrete.

Proof. We claim that $\mu_{n} \stackrel{n \rightarrow \infty}{\longrightarrow} \delta_{\{e\}}$ if and only if $\lim _{n \rightarrow \infty} p_{\mu_{n}}(Q)=1$ for every compact subset $Q \subset G$ such that $e \notin Q$. By definition $\mu_{n} \stackrel{n \rightarrow \infty}{\longrightarrow} \delta_{\{e\}}$ if and only if

$$
\liminf _{n \rightarrow \infty} \mu_{n}(\Omega) \geq \delta_{\{e\}}(\Omega)=1_{\Omega}(\{e\})
$$

for every Chabauty-open subset $\Omega \subset \operatorname{Sub}(G)$. It clearly suffices to consider only such $\Omega$ containing the point $\{e\}$. Observe that $\{e\} \in \mathcal{O}_{1}(Q) \subset \Omega$ for some compact $Q \subset G$ with $e \notin Q$. Since $\mu_{n}\left(\mathcal{O}_{1}(Q)\right)=p_{\mu_{n}}(Q)$ the claim follows. In particular weak-* convergence to $\delta_{\{e\}}$ implies weak triviality.

For the converse direction assume that $\mu_{n}$ is weakly uniformly discrete and weak-* converges to $\delta_{\{e\}}$. Let $Q \subset G$ be any compact subset. If $e \notin Q$ then $\lim _{n \rightarrow \infty} p_{\mu_{n}}(Q)=1$ by the above claim. Finally assume that $e \in Q$ and fix $\varepsilon>0$. By weak uniform discreteness there is an identity neighborhood $U_{\varepsilon}$ so that $p_{\mu_{n}}\left(U_{\varepsilon} \backslash\{e\}\right)>1-\varepsilon$ for all $n \in \mathbb{N}$. Denote $Q_{\varepsilon}=Q \backslash U_{\varepsilon}$ so that $Q_{\varepsilon}$ is compact. Therefore

$$
p_{\mu_{n}}(Q \backslash\{e\}) \geq p_{\mu_{n}}\left(U_{\varepsilon} \backslash\{e\}\right)+p_{\mu_{n}}\left(Q_{\varepsilon}\right)-1 \geq p_{\mu_{n}}\left(Q_{\varepsilon}\right)-\varepsilon \stackrel{n \rightarrow \infty}{\longrightarrow} 1-\varepsilon
$$

The proposition follows by taking $\varepsilon>0$ to be arbitrarily small.

In analogy with Definition 3.3 we say that a sequence $\mu_{n}$ of invariant random subgroups is weakly central if $\lim _{n \rightarrow \infty} p_{\mu_{n}}(Q \backslash Z(G))=1$ for every compact subset $Q \subset G$. Similarly to Proposition 3.4 it is easy to see that any accumulation point of a weakly central sequence is supported on the center, and that the converse holds for weakly uniformly discrete sequences. We remark that dividing a group by its center results in sending a weakly central sequence to a weakly trivial one.

Corollary 3.5. Let $G$ be a semisimple analytic group in zero characteristic. Then a sequence of discrete invariant random subgroups weak-* converging to $\delta_{\{e\}}$ is weakly trivial.

\footnotetext{
${ }^{7}$ Some authors use the terminology Farber for a sequence of lattices $\Gamma_{n}$ so that $\mu_{\Gamma_{n}}$ is trivial in our sense.
} 
We leave the straightforward verification of Example 3.6 to the reader.

Example 3.6. Let $\Gamma$ be a lattice in $G$ and let $\Gamma_{i} \triangleleft \Gamma$ be a descending sequence of normal subgroups with $\bigcap_{i \in \mathbb{N}} \Gamma_{i}=\{e\}$. Then $\Gamma_{i}$ is a weakly trivial sequence.

In particular, the sequence of invariant random subgroups $\mu_{\Gamma_{i}}$ weak-* converges to $\delta_{\{e\}}$ by Proposition 3.4 .

3.3. Quotient spaces and the pointed Gromov-Hausdorff topology. Let $G$ be a l.c.s.c. group. Let $(X, x)$ be a proper locally compact pointed metric space admitting a proper continuous $G$-action by isometries.

Definition 3.7. The space of quotients associated to the $G$-action on $X$ is

$$
\mathcal{Q}(G, X)=\{H \backslash X: H \text { is a closed subgroup of } G\}
$$

The quotients $H \backslash X$ are regarded as pointed metric spaces with basepoints $H x$ and the quotient metrics. $\mathcal{Q}(G, X)$ is equipped with the pointed Gromov-Hausdorff topology.

We will be working with the pointed Gromov-Hausdorff topology in terms of $(\varepsilon, r)$-relations. See [CEG86, I.3.2] for more details on this notion.

Proposition 3.8. The natural map

$$
\operatorname{Sub}(G) \rightarrow \mathcal{Q}(G, X), \quad H \mapsto H \backslash X
$$

is continuous.

Proof. Let $H_{n}$ be a sequence of closed subgroups in $G$ converging to the closed subgroup $H$ in the Chabauty sense. We show that the quotient pointed metric spaces $H_{n} \backslash X$ converge to $H \backslash X$ in the pointed Gromov-Hausdorff topology.

Fix some small $\varepsilon>0$ and large $r>0$. It will suffice to exhibit $(\varepsilon, r)$-relations between $H_{n} \backslash X$ and $H \backslash X$ for all $n$ sufficiently large. Let $\bar{B}(r)$ denote the closed ball $\bar{B}_{X}(x, r)$ in the space $X$. Since $X$ is proper $\bar{B}(r)$ is compact. For every closed subgroup $H$ of $G$ denote

$$
Q_{H}(r)=H \backslash H \bar{B}(r) \subset H \backslash X
$$

so that $Q_{H}(r)$ is compact as well for every $H$. Observe that

$$
Q_{H}(r)=\bar{B}_{H \backslash X}(H x, r)
$$

where $\bar{B}_{H \backslash X}(H x, r)$ is the closed $r$-ball centered at $H x$ in the quotient space $H \backslash X$.

Define a relation $\sim_{n}$ between the compact subsets $Q_{H_{n}}(r)$ and $Q_{H}(r)$ by

$$
H_{n} x \sim_{n} H x, \quad \forall x \in \bar{B}(r)
$$

The relations $\sim_{n}$ need not in general be one-to-one.

We claim that $\sim_{n}$ is an $(\varepsilon, r)$-relation between the two pointed metric spaces $H_{n} \backslash X$ and $H \backslash X$ for all $n$ sufficiently large. Let $d$ and $d_{n}$ denote the induced metrics on the quotient spaces $H \backslash X$ and $H_{n} \backslash X$, respectively. The only non-trivial fact to be proved is that

$$
\left|d_{n}\left(H_{n} x_{1}, H_{n} x_{2}\right)-d\left(H x_{1}, H x_{2}\right)\right|<\varepsilon, \quad \forall x_{1}, x_{2} \in \bar{B}(r)
$$

assuming that $n$ is sufficiently large.

Since $G$ acts properly on $X$ there is a compact subset $C \subset G$ such that $g$. $\bar{B}(3 r) \cap \bar{B}(3 r)=\emptyset$ whenever $g \notin C$. Let $C^{\prime}$ be another compact set containing $C$ 
in its interior. The Chabauty convergence of $H_{n}$ to $H$ implies that for every open identity neighborhood $U \subset G$ we have that

$$
H_{n} \cap C \subset(H \cap C) U \text { and } H \cap C \subset\left(H_{n} \cap C^{\prime}\right) U
$$

for all $n$ sufficiently large. Note that

$$
d\left(H x_{1}, H x_{2}\right)=\min _{h \in H \cap C} d\left(x_{1}, h x_{2}\right)=\min _{h \in H \cap C^{\prime}} d\left(x_{1}, h x_{2}\right)
$$

and a similar expression holds true for $d_{n}\left(H_{n} x_{1}, H_{n} x_{2}\right)$ and every $n \in \mathbb{N}$. Thus (1) follows from the continuity of the $G$-action and the compactness of $C^{\prime}$.

We deduce:

Corollary 3.9. The space $\mathcal{Q}(G, X)$ is compact.

Since $G$ is second countable it admits a left-invariant proper metric. Regard $G$ as a pointed metric space with basepoint at the identity. Therefore the space of quotients $\mathcal{Q}(G, G)$ is well defined and consists of the quotients $H \backslash G$ where $H$ is a closed subgroup of $G$. The mapping $\operatorname{Sub}(G) \ni H \mapsto H \backslash G$ is continuous. However, note that in general it need not be injective.

A generalization of the previous construction is obtained as follows. Assume that the metric on $G$ is right- $K$-invariant for some closed subgroup $K$ in $G$. For instance, this is always possible when $K$ is compact. The space of quotients $\mathcal{Q}(G, G / K)$ is well defined and consists of the double coset spaces $H \backslash G / K$ where $H \in \operatorname{Sub}(G)$.

Remark 3.10. The space of graphs $\mathcal{G R}(G, X)$ consists of the graphs of the quotient maps $X \rightarrow H \backslash X$ regarded as subspaces of $X \times H \backslash X$. These are pointed metric spaces with basepoints $(x, H x)$. The space $\mathcal{G R}(G, X)$ is equipped with the pointed Gromov-Hausdorff topology. There is a pair of maps

$$
\operatorname{Sub}(G) \rightarrow \mathcal{G R}(G, X) \rightarrow \mathcal{Q}(G, X)
$$

whose composition is the map considered in Proposition 3.8. A small modification of the proof of Proposition 3.8 shows that the two intermediate maps are continuous as well.

The point of this construction is that the map $\operatorname{Sub}(G) \rightarrow \mathcal{G R}(G, X)$ is obviously bijective and is therefore a homeomorphism. In particular the topology on $\mathcal{G R}(G, X)$ does not depend on the particular choice of the proper metric on $X$. In a vague sense, the topology of $\mathcal{Q}(G, X)$ is also independent of the metric, but the fibers of the map from $\operatorname{Sub}(G)$ may depend on the choice of metric.

We will not be making use of the space of graphs in this work.

3.4. Benjamini-Schramm convergence. Let the group $G$ and the pointed metric space $X$ be as above. The Benjamini-Schramm space $\mathcal{B S}(G, X)$ is the space of Borel probability measures on $\mathcal{Q}(G, X)$ with the weak-* topology. Since the space of quotients is compact so is the Benjamini-Schramm space. Moreover, in view of Proposition 3.8 we have:

Corollary 3.11. The natural map $\operatorname{IRS}(G) \rightarrow \mathcal{B S}(G, X)$ is continuous.

The natural map of Corollary 3.11 takes a sequence in IRS $(G)$ converging to $\delta_{\{e\}}$ to a sequence in $\mathcal{B S}(G, X)$ converging to $\delta_{X}$.

The following proposition provides a geometric interpretation for this convergence in the special case of semisimple analytic groups. 
Proposition 3.12. Let $G$ be a semisimple analytic group and $X$ the corresponding product of a symmetric space and a Bruhat-Tits building. Every weak-* accumulation point of the sequence $\mu_{n} \in \operatorname{IRS}(G)$ is central if and only if

$$
\operatorname{Prob}_{\mu_{n}}\left(B_{H \backslash X}(H x, r) \text { is isometric to } B_{X}(x, r)\right) \stackrel{n \rightarrow \infty}{\longrightarrow} 1
$$

for every radius $0<r<\infty$. Here $x \in X$ is an arbitrary basepoint.

Proof. Write $G=C \times D$ where $C$ and $D$ are the Archimedean and non-Archimedean factors of $G$, respectively. For every fixed radius $r>0$ denote $B_{r}=\bar{B}_{X}(x, r)$ and consider the subset

$$
Q_{r}=\left\{g \in G: g B_{r} \cap B_{r} \neq \emptyset\right\} \subset G .
$$

The properness of the action implies that $Q_{r}$ is compact. Consider the subset

$$
V_{r}=\left\{g \in D: g_{\mid B_{r}}=\operatorname{id}_{\mid B_{r}}\right\} .
$$

Note that $V_{r}$ is a compact open subgroup in $D$. Let $U_{r} \subset C$ be a neighborhood of $Z(C)$ so that the only closed subgroups of $C$ contained in $U_{r}$ are central and such that $U_{r} \times V_{r} \subset Q_{r}$. Assume that $U_{r^{\prime}} \subset U_{r}$ for every $r^{\prime}>r$ and $\bigcap_{r} U_{r}=Z(C)$.

Consider the Chabauty open subset $\Omega_{r}=\mathcal{O}_{1}\left(Q_{r} \backslash\left(U_{r} \times V_{r}\right)\right)$. A closed subgroup $H$ belongs to $\Omega_{r}$ if and only if $H \cap Q_{r} \subset V_{r}$ if and only if the $r$-balls at the basepoints of $H \backslash X$ and of $X$ are isometric. Just as in the proof of Proposition 3.4 the fact that every accumulation point of $\mu_{n}$ is central is equivalent to $\lim _{n \rightarrow \infty} p_{\mu_{n}}(Q)=1$ for every compact subset $Q \subset G$ with $Z(G) \cap Q=\{e\}$. Every such compact subset $Q$ is contained in $Q_{r} \backslash\left(U_{r} \times V_{r}\right)$ for all $r$ sufficiently large, and the result follows.

In the situation of Proposition 3.12 the probability with respect to $\mu_{n}$ that a random $r$-ball in $H \backslash X$ is contractible tends to one for every radius $0<r<\infty$.

\section{On Zariski Closure and the Chabauty topology}

Let $k$ be any local field. We analyze the Zariski closure operation on the Chabauty space of closed subsets in the group $\mathbb{G}(k)$ of $k$-rational points of an algebraic $k$-group $\mathbb{G}$. As an application we define a certain variant - local Zariski closure operation - which turns out to be useful in positive characteristic.

The section begins with a rather general discussion on $k$-vector spaces. As we progress we specialize to study the linear representation of the group $\mathbb{G}(k)$ in the $k$-linear space of its regular functions arising from the action of $\mathbb{G}$ on itself by conjugation. Some of our arguments are inspired by [AB94, §4].

4.1. Limits of finite dimensional vector spaces. Consider a sequence $A_{i}$ of finite dimensional $k$-vector spaces for $i \in \mathbb{N}$. Assume that this sequence admits a pair of compatible structures as a direct and an inverse system. That is, there are $k$-linear maps $\iota_{i}: A_{i} \rightarrow A_{i+1}$ and $\sigma_{i}: A_{i+1} \rightarrow A_{i}$ that satisfy $\sigma_{i} \circ \iota_{i}=\operatorname{id}_{\mid A_{i}}$ for all $i \in \mathbb{N}$. In particular $\iota_{i} \circ \sigma_{i}$ is an idempotent $k$-endomorphism of $A_{i+1}$ for every $i \in \mathbb{N}$.

The Grassmannian $\mathcal{G R}\left(A_{i}\right)$ is by definition the disjoint union of its $d$-dimensional parts with $0 \leq d \leq \operatorname{dim} A_{i}$ for every $i \in \mathbb{N}$. We obtain a compatible direct and inverse system structures on the sequence $\mathcal{G} \mathcal{R}\left(A_{i}\right)$ of Grassmannians. We retain the notations $\iota_{i}$ and $\sigma_{i}$ for the induced maps in these systems.

Let $A$ denote the $k$-vector space direct limit $A=\underline{\lim }_{i} A_{i}$. The Grassmannian $\mathcal{G R}(A)$ consists of all the $k$-vector subspaces of $A$ and it is easy to verify that 
$\mathcal{G R}(A)=\lim _{i} \mathcal{G R}\left(A_{i}\right)$. Since $k$ is a local field every $\mathcal{G R}\left(A_{i}\right)$ is compact. In particular $\mathcal{G R}(A)$ becomes a compact space with the inverse limit topology.

The dual $k$-vector space of $A$ is the inverse limit $A^{*}=\lim _{i} A_{i}^{*}$. This is a topological space with the inverse limit topology. As before let $\mathcal{G} \mathcal{R}\left(A^{*}\right)$ denote the Grassmannian of all $k$-vector subspaces of $A^{*}$. There is an order-reversing bijection

$$
\text { ann }: \mathcal{G R}\left(A^{*}\right) \rightarrow \mathcal{G R}(A)
$$

obtained by taking annihilators. We use the notation $\iota_{i}^{*}$ and $\sigma_{i}^{*}$ for the maps in the dual inverse and direct systems, as well as the induced maps in the system $\mathcal{G} \mathcal{R}\left(A_{i}^{*}\right)$.

4.2. The linear span map. Consider the linear span map denoted sp and defined on the power set of $A^{*}$ by

$$
\text { sp : } \operatorname{Pow}\left(A^{*}\right) \rightarrow \mathcal{G R}\left(A^{*}\right), \quad \operatorname{sp}(F)=\operatorname{span}_{k}(F) \quad \forall F \subset A^{*} .
$$

Given a topological space $X$ we let $\mathrm{Cl}(X)$ denote the space of closed subsets of $X$ regarded with the Chabauty topology, as in Definition 3.1

Proposition 4.1. Let $X$ be a topological space and $\varepsilon: X \rightarrow A^{*}$ a continuous map. Denote by $\hat{\varepsilon}$ the corresponding set map $\mathrm{Cl}(X) \rightarrow \operatorname{Pow}\left(A^{*}\right)$. Then the composition

$$
\text { ann } \circ \mathrm{sp} \circ \hat{\varepsilon}: \mathrm{Cl}(X) \rightarrow \operatorname{Pow}\left(A^{*}\right) \rightarrow \mathcal{G R}\left(A^{*}\right) \rightarrow \mathcal{G R}(A)
$$

is Borel measurable.

Note that we have not explicitly defined a Borel structure on the two intermediate spaces $\operatorname{Pow}\left(A^{*}\right)$ or on $\mathcal{G} \mathcal{R}\left(A^{*}\right)$.

Proof. It will suffice to show that the composition of ann $\circ$ sp $\circ \hat{\varepsilon}$ with the projection $\mathcal{G R}(A) \rightarrow \mathcal{G R}\left(A_{i}\right)$ is upper semi-continuous, and in particular Borel measurable, for every $i \in \mathbb{N}$. This composition, denoted $\alpha_{i}$, is equal to

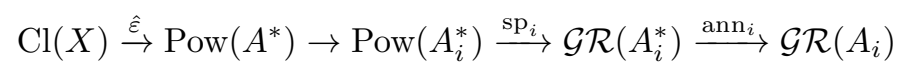

where the second map is induced from the projection $A^{*} \rightarrow A_{i}^{*}, \mathrm{sp}_{i}$ is the $k$-linear span map on $A_{i}^{*}$ and $\operatorname{ann}_{i}$ is the annihilator map between $\mathcal{G R}\left(A_{i}^{*}\right)$ and $\mathcal{G R}\left(A_{i}\right)$ which is an order-reversing homeomorphism.

We need to show that $\alpha_{i}$ is lower semi-continuous. Consider a closed subset $F \subset$ $X$ with $\operatorname{dim} \alpha_{i}(F)=d$ where $0 \leq d \leq \operatorname{dim} A_{i}^{*}$. There are $d$ points $x_{1}, \ldots, x_{d} \in F$ such that

$$
\alpha_{i}(F)=\operatorname{sp}_{i} \circ \hat{\varepsilon}\left(\left\{x_{1}, \ldots, x_{d}\right\}\right) .
$$

The continuity of the map $\varepsilon$ implies that the subspace $\operatorname{sp}_{i} \circ \hat{\varepsilon}\left(\left\{x_{1}^{\prime}, \ldots, x_{d}^{\prime}\right\}\right)$ is close to $\alpha_{i}(F)$ in the topology of $\mathcal{G R}\left(A_{i}^{*}\right)$. So these two subspaces have the same dimension $d$ for points $x_{i}^{\prime}$ sufficiently close to $x_{i}$ in $X$. This determines a Chaubuty neighborhood $\Omega$ of $F$ so that $\alpha_{i}\left(F^{\prime}\right) \subset \operatorname{sp}_{i} \circ \hat{\varepsilon}\left(\left\{x_{1}^{\prime}, \ldots, x_{d}^{\prime}\right\}\right)$ for $F^{\prime} \in \Omega$ as required.

4.3. Regular functions on affine spaces. We specialize the discussion to our case of interest, which is the algebra of $k$-regular functions on an affine space.

Consider the algebra $A$ of $k$-regular functions on the $N$-dimensional affine space

$$
A=k\left[x_{1}, \ldots, x_{N}\right]
$$

for some fixed $N \in \mathbb{N}$. For our purposes $A$ is regarded simply as an $k$-vector space, and it is in fact the direct limit of the finite dimensional $k$-vector spaces

$$
A_{i}=\left\{p \in k\left[x_{1}, \ldots, x_{N}\right]: \operatorname{deg} p \leq i\right\}
$$


with the direct system of maps $\iota_{i}: A_{i} \rightarrow A_{i+1}$ being the inclusion. An inverse system of maps $\sigma_{i}: A_{i+1} \rightarrow A_{i}$ is given by

$$
p=\sigma_{i}(p)+r, \quad \text { s.t. } \sigma_{i}(p) \in A_{i} \text { and } r \text { has no non-zero monomials of degree } \leq i
$$

for all $i \in \mathbb{N}$ and polynomials $p \in A_{i+1}$. The two systems $\iota_{i}$ and $\sigma_{i}$ are compatible in our previous sense, that is $\sigma_{i} \circ \iota_{i}=\mathrm{id}_{\mid A_{i}}$.

Let $\mathrm{I}(F)$ denote the ideal in $A$ of vanishing $k$-regular functions on the subset $F$ of the affine space $k^{N}$. In other words

$$
\mathrm{I}(F)=\{p \in A: p(x)=0 \quad \forall x \in F\} .
$$

By definition $\mathrm{I}(F)=\mathrm{I}\left(\bar{F}^{\mathrm{Z}}\right)$ where $\bar{F}^{\mathrm{Z}}$ is the Zariski closure of $F$ in $k^{N}$.

Proposition 4.2. Consider $k^{N}$ with the topology arising from the local field $k$. Then the map I : $\mathrm{Cl}\left(k^{N}\right) \rightarrow \mathcal{G} \mathcal{R}(A)$ is Borel measurable.

Proof. The evaluation map $\varepsilon: k^{N} \rightarrow A^{*}$ is defined by

$$
\varepsilon(x)(p)=p(x) \quad \forall x \in k^{N}, p \in A .
$$

Extend $\varepsilon$ to a set map $\hat{\varepsilon}: \mathrm{Cl}\left(k^{N}\right) \rightarrow \operatorname{Pow}\left(A^{*}\right)$. Observe the identity

$$
\mathrm{I}=\operatorname{ann} \circ \operatorname{sp} \circ \hat{\varepsilon}: X \rightarrow \mathcal{G R}(A) .
$$

The fact that $I$ is Borel measureable follows from Proposition 4.1

The proof of Proposition 4.1 gives more information, namely that the composition of I with a projection $\mathcal{G R}(A) \rightarrow \mathcal{G R}\left(A_{i}\right)$ is upper semi-continuous.

4.4. Actions with fixed points on affine spaces. Let $\mathrm{GL}(A)$ denote the group of all $k$-linear automorphisms of $A$. GL $(A)$ admits a subgroup $\mathrm{GL}^{*}(A)$ given by

$$
\mathrm{GL}^{*}(A)=\left\{\alpha \in \mathrm{GL}(A): \sigma_{i} \alpha=\sigma_{i} \alpha \sigma_{i} \quad \forall i \in \mathbb{N}\right\}
$$

where $\sigma_{i}$ is the map $A \rightarrow A_{i}$ and $A_{i}$ is considered as a subspace of $A$. The subgroups $\mathrm{GL}^{*}\left(A_{i}\right)$ of $\mathrm{GL}\left(A_{i}\right)$ may be analogously defined for every $i \in \mathbb{N}$. For $\alpha \in \mathrm{GL}^{*}(A)$ we set $\alpha_{i}=\sigma_{i} \alpha \in \mathrm{GL}^{*}\left(A_{i}\right)$ for every $i \in \mathbb{N}$. Observe that $\mathrm{GL}^{*}(A)=\lim _{i} \mathrm{GL}^{*}\left(A_{i}\right)$.

Let $G$ be any group admitting an action on the $N$-dimensional affine space defined over $k$. We obtain a representation $\tau$ of $G$ in the $k$-vector space $A$. Namely $\tau$ is a group homomorphism

$$
\tau: G \rightarrow \operatorname{GL}(A), \quad \tau(g)(p)=p \circ f_{g^{-1}}, \quad \forall p \in A
$$

so that for every element $g \in G$ the map $f_{g}=\left(f_{g}^{1}, \ldots, f_{g}^{N}\right)$ is an automorphism from $k^{N}$ to itself given by $k$-polynomials.

Proposition 4.3. If the action of $G$ fixes the point $0 \in k^{N}$ then $\tau(G) \subset \mathrm{GL}^{*}(A)$.

Proof. We need to verify that $\sigma_{i} \tau(g) \sigma_{i}=\sigma_{i} \tau(g)$ holds for every element $g \in G$ and every $i \in \mathbb{N}$. The fixed point assumption means that the polynomials $f_{g}^{j}$ have a vanishing constant term for every element $g \in G$ and every index $1 \leq j \leq N$, and this implies the required condition.

The projectivization of the representation $\tau$ gives rise to a natural action $\bar{\tau}$ of $G$ on the Grassmannian $\mathcal{G R}(A)$. 
4.5. The conjugation action of an algebraic group. We further specialize our discussion to the conjugation action of an algebraic group on itself.

Let $\mathbb{G}$ be a linear group defined over $k$. We may assume that $\mathbb{G}$ is affine Bor12, 1.10] and that $\mathbb{G} \leq \mathrm{GL}_{M}$ for some $M \in \mathbb{N}$. Denote $G=\mathbb{G}(k)$ so that

$$
G \subset \mathrm{M}_{M}(k) \cong k^{N} \quad \text { where } N=M^{2} .
$$

Consider the action of $G$ on $\mathrm{M}_{M}(k) \cong k^{N}$ by matrix conjugation. This action is defined over $k$ and fixes zero. There is a corresponding representation $\tau$ of the group $G$ in the the $k$-vector space $A=k\left[x_{1}, \ldots, x_{N}\right]$. According to Proposition 4.3 in fact $\tau(G) \subset \mathrm{GL}^{*}(A)$ and $\tau=\lim _{\leftarrow} \tau_{i}$ for a family of representations $\tau_{i}: G \rightarrow \mathrm{GL}^{*}\left(A_{i}\right)$. The representations $\tau_{i}$ are continuous.

Definition 4.4. The map $\mathrm{I}_{G}: \operatorname{Sub}(G) \rightarrow \mathcal{G R}(A)$ is the composition

$$
\mathrm{I}_{G}: \operatorname{Sub}(G) \rightarrow \mathrm{Cl}\left(k^{N}\right) \stackrel{\mathrm{I}}{\rightarrow} \mathcal{G R}(A)
$$

where the map I is given prior to Proposition 4.2 above.

Clearly $\mathrm{I}_{G}(G) \subset \mathrm{I}_{G}(F)$ for every closed subset $F$ in $G$. Moreover, for every closed subgroup $H$ in $G$ the ideal $\mathrm{I}_{G}(H) \triangleleft A$ corresponds to its Zariski closure $\bar{H}^{\mathrm{Z}}$. Of course, $\bar{H}^{\mathrm{Z}}$ is a Zariski closed subgroup of $G$. The map $\mathrm{I}_{G}$ is $G$-equivariant with respect to the conjugation action on $\operatorname{Sub}(G)$ and the $\bar{\tau}$-action on $\mathcal{G R}(A)$.

4.6. Intersections of conjugates. As an application of the above discussion, we obtain a result on the intersection of finitely many conjugates of a Zarsiki closed subgroup. This result will be later used in the proof of Lemma 7.7

Proposition 4.5. Let $N$ and $H$ be a pair of Zariski closed subgroups in $G$ so that $N \leq H$ and $N \triangleleft G$. Let $\Gamma \leq G$ be a Zariski dense subgroup. Assume that

$$
\bigcap_{\gamma \in \Gamma} H^{\gamma}=N
$$

Then there is some $m \in \mathbb{N}$, elements $\gamma_{1}, \ldots, \gamma_{m} \in \Gamma$ and an identity neighborhood $W$ in $G$ so that

$$
\bigcap_{n=1}^{m} H^{\gamma_{n} w_{n}}=N
$$

holds for every choice of elements $w_{n} \in W$.

For the proof recall that $\bar{\tau}$ is the representation of $G$ on the Grassmaniann space $\mathcal{G R}(A)$ arising from $\tau$. In fact $\bar{\tau}=\lim _{\longleftarrow} \bar{\tau}_{i}$ where $\bar{\tau}_{i}$ are the projective actions of $G$ on the Grassmanianns $\mathcal{G} \mathcal{R}\left(A_{i}\right)$ arising from the finite dimensional $\tau_{i}$ 's.

Proof of Proposition 4.5. Denote $I_{H}=\mathrm{I}_{G}(H)$. This is a point in $\mathcal{G R}(A)$ corresponding to an ideal in $A=\lim _{i \in \mathbb{N}} A_{i}$. Consider the ideal

$$
J=\sum_{\gamma \in \Gamma} \bar{\tau}(\gamma) I_{H} \in \mathcal{G} \mathcal{R}(A) .
$$

Clearly $J$ is a $\bar{\tau}(\Gamma)$-invariant subspace of $A$. By assumption $J$ determines the Zariski closed subgroup $N$. The Noetherianity of $A$ implies that $J$ admits a finite generating set $S$ and that moreover $J=\sum_{n=1}^{m} \bar{\tau}\left(\gamma_{n}\right) I_{H}$ for some $m \in \mathbb{N}$ and elements $\gamma_{1}, \ldots, \gamma_{m} \in \Gamma$. 
Choose a sufficiently large index $i \in \mathbb{N}$ so that $S \subset A_{i}$ and let $\sigma_{i}$ denote the natural projection $\mathcal{G} \mathcal{R}(A) \rightarrow \mathcal{G} \mathcal{R}\left(A_{i}\right)$. Observe that

$$
S=\sigma_{i}(S) \subset \sigma_{i}(J)=\sum_{n=1}^{m} \bar{\tau}_{i}\left(\gamma_{n}\right) \sigma_{i}\left(I_{H}\right) .
$$

The projective representation $\bar{\tau}_{i}$ is continuous. Since $\sigma_{i}(J)$ is $\bar{\tau}_{i}(\Gamma)$-invariant the classical Borel density theorem [Mar91, II.4.4] implies that $\sigma_{i}(J)$ is $\bar{\tau}_{i}(G)$-invariant as well. Therefore there is a sufficiently small identity neighborhood $W \subset G$ so that the above condition continues to hold up to passing to elements of the form $g_{n} w_{n}$ with $w_{n} \in W$. This shows that the ideal $I^{\prime}=\sum_{n=1}^{m} \bar{\tau}\left(\gamma_{n} w_{n}\right) I_{H}$ contains the generating set $S$ and in particular that $J=I^{\prime}$. The required conclusion follows.

4.7. Local Zariski closure. A certain modification $\mathrm{J}_{G}$ of the map $\mathrm{I}_{G}$ introduced above allows us to use Zariski closure in a local manner. Fix a countable local basis $U_{i}$ of neighborhoods at the identity for $G$.

Definition 4.6. The map $\mathrm{J}_{G}$ is given by

$$
\mathrm{J}_{G}: \operatorname{Sub}(G) \rightarrow \mathcal{G} \mathcal{R}(A), \quad \mathrm{J}_{G}(H)=\operatorname{span}_{i} \mathrm{I}\left(H \cap \bar{U}_{i}\right)
$$

Note that this definition is independent of the particular choice of a local basis. Our treatment of the map $\mathrm{I}_{G}$ applies to the map $\mathrm{J}_{G}$ as well. In particular, it follows from Proposition 4.1 that $\mathrm{J}_{G}$ is Borel measurable and $G$-equivariant for the corresponding actions.

Proposition 4.7. Let $G$ be a semisimple analytic group over the local field $k$ and $H$ a closed subgroup in $G$.

(1) If $k$ is Archimedean then $\mathrm{J}_{G}(H)=\mathrm{I}_{G}\left(H_{0}\right)$, and

(2) If $k$ is non-Archimedean then there is a compact open subgroup $K_{H} \leq G$ depending on $H$ so that $\mathrm{J}_{G}(H)=\mathrm{I}_{G}\left(H \cap K_{H}\right)$.

Proof. (11) In the Archimedean case the connected component at the identity $H_{0}$ is open in $H$ and therefore $\mathrm{J}_{G}(H)=\mathrm{J}_{G}\left(H_{0}\right)$. In zero characteristic, a subset of an irreducible variety which is open in the Hausdorff $k$-topology is Zariski dense PR92, Lemma 3.2]. In particular $\mathrm{J}_{G}\left(H_{0}\right)=\mathrm{I}_{G}\left(H_{0}\right)$.

(2) Noetherianity implies that there is an identity neighborhood $U_{H}$ in $G$ depending on $H$ so that $\mathrm{J}_{G}(H)=\mathrm{I}\left(H \cap \overline{U_{H}}\right)$. In the non-Archimedean case we may assume that $K_{H}=\overline{U_{H}}$ is a compact open subgroup.

Note that in the non-Archimedean case $\mathrm{J}_{G}(H)=\mathrm{I}_{G}\left(H \cap K_{H}^{\prime}\right)$ as well for every compact open subgroup $K_{H}^{\prime}$ contained in $K_{H}$.

4.8. Totally disconnected factors and local Zariski closure. Given any totally disconnected l.c.s.c. group $D$ the definition of the map $\mathrm{J}_{G}$ can be generalized by extending its domain to the product Chabauty space $\operatorname{Sub}(G \times D)$. This will be useful in the proof of Theorem 1.9 in $\$ 5$ below. Denote $G_{1}=G \times D$ and fix a countable local basis of identity neighborhoods $V_{i}$ for $G_{1}$.

Definition 4.8. The map $\mathrm{J}_{G_{1}, G}$ is given by

$$
\mathrm{J}_{G_{1}, G}: \operatorname{Sub}\left(G_{1}\right) \rightarrow \mathcal{G R}(A), \quad \mathrm{J}_{G_{1}, G}(H)=\operatorname{span}_{i} \mathrm{I}\left(\operatorname{pr}_{G}\left(H \cap \bar{V}_{i}\right)\right)
$$

Here $\operatorname{pr}_{G}$ is the projection map $G_{1}=G \times D \rightarrow G$. 
Our discussion of the map $\mathrm{J}_{G}$ naturally extends to the map $\mathrm{J}_{G_{1}, G}$. In particular this map is measurable and equivariant. If $G$ itself is non-Archimedean then an analogue of Proposition 4.7 holds true for $\mathrm{J}_{G_{1}, G}$ in the sense that every closed subgroup $H \leq G_{1}=G \times D$ satisfies $\mathrm{J}_{G_{1}, G}(H)=\mathrm{I}_{G}\left(\operatorname{pr}_{G}\left(H \cap K_{H}\right)\right)$ with some compact open subgroup $K_{H} \leq G_{1}$ depending on $H$.

\section{BorEl DENSITY THEOREM FOR INVARIANT RANDOM SUBGROUPS}

Fix a local field $k$ and let $G$ a happy semisimple analytic group over $k$. We prove a result on invariant random subgroups in $G$ generalizing the classical Borel density theorem. The main difficulty is in dealing with the positive characteristic case relying on the methods of $\$ 4$ and on Pink's theorem.

5.1. Invariant probability measures on Grassmannians. Morally, the key idea is coming from Furstenberg's proof of Borel's theorem [Fur76.

Proposition 5.1 (Furstenberg's lemma). Let $V$ be a finite dimensional $k$-vector space. Let $\rho: G \rightarrow \mathrm{GL}(V)$ a k-rational representation and $\bar{\rho}: G \rightarrow \operatorname{PGL}(V)$ the corresponding projective representation. Then every $\bar{\rho}\left(G^{+}\right)$-invariant probability measure on $\mathrm{P}(V)$ is supported on $\bar{\rho}\left(G^{+}\right)$-invariant points.

Proof. The analogous statement is established in [Fur76, Lemma 3] assuming that $G$ is a minimally almost periodic topological group, i.e. $G$ has no non-trivial continuous homomorphisms into compact groups. This assumption is used twice throughout the proof, and we indicate alternative arguments in our case. We restrict attention to the subgroup $G^{+}$.

(1) $\rho\left(G^{+}\right)$is not relatively compact in $\operatorname{GL}(V)$. Since $G$ is $k$-isotropic it contains some $k$-split torus $S$. The image $\rho(S)$ is $k$-split as well [Bor12, 8.2] and is in particular not relatively compact. Since $G^{+}$is cocompact in $G$ the image $\rho\left(S \cap G^{+}\right)$is already not relatively compact in $\mathrm{GL}(\mathrm{V})$.

(2) $G^{+}$has no non-trivial homomorphisms into finite groups. Indeed it is known that the group $G^{+}$has no finite index subgroups [Mar91, I.2.3.2].

We can now proceed verbatim as in Fur76, Lemma 3] and deduce that a $\bar{\rho}\left(G^{+}\right)$invariant probability measure on $P(V)$ is supported on $\bar{\rho}\left(G^{+}\right)$-invariant points.

Assume that $G$ admits a $k$-action on the $N$-dimensional affine space. This gives a representation $\tau: G \rightarrow \mathrm{GL}(A)$ where $A=k\left[x_{1}, \ldots, x_{N}\right]$. Recall from $\$ 4$ that $A$ is a $k$-vector space direct limit $A=\lim _{i \in \mathbb{N}} A_{i}$. The finite dimensional $k$-vector spaces $A_{i}$ admit a direct system $\iota_{i}$ as well as a compatible inverse system $\sigma_{i}$.

Assume moreover that $0 \in k^{N}$ is a fixed point for the $G$-action. In that case it follows from Proposition 4.3 that $\tau(G) \subset \mathrm{GL}^{*}(A)$ and $\tau=\lim _{i} \tau_{i}$ with $\tau_{i}: G \rightarrow$ $\mathrm{GL}^{*}\left(A_{i}\right)$. Let $\bar{\tau}$ denote the representation of $G$ on the Grassmaniann space $\mathcal{G} \mathcal{R}(A)$ arising from $\tau$. We obtain $\bar{\tau}=\lim \bar{\tau}_{i}$ where $\bar{\tau}_{i}$ is an action of $G$ on the Grassmaniann $\mathcal{G R}\left(A_{i}\right)$ arising from $\tau_{i}$.

Proposition 5.2. Let $\mu$ be a $\bar{\tau}\left(G^{+}\right)$-invariant probability measure on $\mathcal{G} \mathcal{R}(A)$. If $0 \in k^{N}$ is a $G$-fixed point then $\mu$ is supported on $\bar{\tau}\left(G^{+}\right)$-fixed points.

Proof. A standard ergodic decomposition argument allows us to assume without loss of generality that $\mu$ is ergodic with respect to $\bar{\tau}\left(G^{+}\right)$.

Pushing forward $\mu$ by means of the inverse system of maps $\mathcal{G} \mathcal{R}(A) \rightarrow \mathcal{G R}\left(A_{i}\right)$ we obtain ergodic $\bar{\tau}_{i}\left(G^{+}\right)$-invariant probability measures $\mu_{i}$ on $\mathcal{G} \mathcal{R}\left(A_{i}\right)$ for every 
$i \in \mathbb{N}$. Ergodicity implies that there are numbers $0 \leq d_{i} \leq \operatorname{dim} A_{i}$ so that $\mu_{i}$ is supported on the $d_{i}$-dimensional part of $\mathcal{G R}\left(A_{i}\right)$.

Let $\rho_{i}=\wedge^{d_{i}} \tau_{i}$ denote the wedge representation of $G^{+}$in $V_{i}=\wedge^{d_{i}} A_{i}$ and $\bar{\rho}_{i}$ the projectivization of $\rho_{i}$. The Plücker embedding allows us to identify the $d_{i^{-}}$ dimensional part of the Grassmannian $\mathcal{G} \mathcal{R}\left(A_{i}\right)$ with a subset of the projective space $\mathrm{P}\left(V_{i}\right)$. Under this identification $\mu_{i}$ can be regarded as a $\bar{\rho}_{i}\left(G^{+}\right)$-invariant probability measure on $\mathrm{P}\left(V_{i}\right)$. By Furstenberg's lemma and since $\mu_{i}$ is ergodic it must be supported on a single point corresponding to a $d_{i}$-dimensional subspace $I_{i} \leq A_{i}$.

Passing to the inverse limit we obtain that $\mu$ is an atomic point mass supported on some $\tau\left(G^{+}\right)$-fixed point $I \in \mathcal{G R}(A)$.

5.2. Proof of the Borel density theorem for IRS. We present a proof of Theorem 1.9 relying on the above version of Furstenberg's lemma as well as Pink's theorem. We start with some preparation.

Proposition 5.3. Let $G^{\dagger}$ be an intermediate subgroup $G^{+} \leq G^{\dagger} \leq G$. Let $F$ be an almost direct factor of $G$. Let $\mu$ be an ergodic invariant random subgroup of $G^{\dagger}$. Then there is a pair of Zariski closed normal subgroups $N, M \triangleleft G$ with $N \leq M$ so that

- in case $k$ is Archimedean $N={\overline{H_{0}}}^{Z}$,

- in case $k$ is non-Archimedean $N={\overline{\mathrm{pr}_{F}\left(H \cap K_{H}\right)}}^{\mathrm{Z}}$ for some compact open subgroup $K_{H}$ depending on $H$, and

- $M=\bar{H}^{\mathrm{Z}}$ in both cases

for $\mu$-almost every closed subgroup $H$ in $G^{\dagger}$.

Here $\operatorname{pr}_{F}$ denotes the natural projection $G \rightarrow F$.

Proof. We first deal with Zariski closure and the normal subgroup $M$.

Recall the map $\mathrm{I}_{G}: \operatorname{Sub}(G) \rightarrow \mathcal{G R}(A)$ introduced in $\$ 4$. Given a closed subgroup $H$ in $G$ the point $\mathrm{I}_{G}(H)$ corresponds to the ideal of vanishing regular functions associated with the Zariski closure $\bar{H}^{\mathrm{Z}}$ and regarded as a $k$-linear subspace of $A$.

Consider $\mu$ as a probability measure on $\operatorname{Sub}(G)$ and let $\nu$ denote the pushforward ergodic $\bar{\tau}\left(G^{\dagger}\right)$-invariant probability measure on $\mathcal{G} \mathcal{R}(A)$ given by $\nu=\left(\mathrm{I}_{G}\right)_{*} \mu$.

The measure $\nu$ is supported on $\bar{\tau}\left(G^{+}\right)$-invariant points in $\mathcal{G R}(A)$ according to Proposition 5.2. Every $\bar{\tau}\left(G^{+}\right)$-invariant point $I \in \mathcal{G R}(A)$ satisfies $\mathrm{I}_{G}(G) \leq I$ and corresponds to a Zariski closed subgroup of $G$ normalized by $G^{+}$. Such points are in fact $\bar{\tau}(G)$-invariant, by Proposition 2.3. The ergodicity of $\nu$ implies that $\bar{H}^{\mathrm{Z}}=M$ for some normal subgroup $M \triangleleft G$ and for $\mu$-almost every $H$.

The conclusion involving the subgroup $N$ follows in an analogous manner relying on the map $\mathrm{J}_{G}: \operatorname{Sub}(G) \rightarrow \mathcal{G R}(A)$ and Proposition 4.7

In the non-Archimedean case and taking into account the factor $F$ we rely on the map $\mathrm{J}_{G, F}$ as in Definition 4.8 and the remarks following it. In that case Proposition 5.2 is applied with respect to the simple analytic group $F$.

The trace field $\mathcal{T} \mathcal{F}_{\rho}$ of a $k$-representation $\rho$ was introduced in Definition 2.6.

Proposition 5.4. Let $G^{\dagger}$ be an intermidiate subgroup $G^{+} \leq G^{\dagger} \leq G$ and $\mu$ an ergodic invariant random subgroup of $G^{\dagger}$. Let $S$ be an adjoint absolutely simple factor of $G$ and $\rho$ a k-representation of $S$. 
If $\mathrm{J}_{G, S}(H)=\mathrm{I}_{S}(S)$ for $\mu$-almost every closed subgroup $H$ then for every compact open subgroup $U$ of $G$ the trace field $\mathcal{T F}_{\rho}\left(\operatorname{pr}_{S}(H \cap U)\right)$ is $\mu$-almost everywhere constant.

As usual $\mathrm{pr}_{S}$ denotes the projection $G \rightarrow S$. The notation $\mathrm{J}_{G, S}$ is introduced in Definition 4.8. Note that the essential value of the trace field does not depend on the choice of the compact open subgroup $U$.

Proof. Let Sub $\left(k_{+}\right)$denote the Chabauty space of the local field $k$ regarded as an additive group. We claim that the following map $\mathcal{T}$

$$
\mathcal{T}: \operatorname{Sub}(G) \rightarrow \operatorname{Sub}(S) \rightarrow \operatorname{Sub}\left(k_{+}\right), \quad \Gamma \mapsto \operatorname{pr}_{S}(\Gamma \cap U) \mapsto \mathcal{T} \mathcal{F}_{\rho}\left(\operatorname{pr}_{S}(\Gamma \cap U)\right)
$$

is Borel measurable. Recall that the Chabauty Borel structure is generated by subsets of the form $O_{2}(U)$ in the sense of Definition 3.1. It follows immediately that the map $\operatorname{Sub}(G) \rightarrow \operatorname{Sub}(S)$ as above is Borel measurable.

It remains to verify that the map taking a closed subgroup $L$ of $S$ to its trace field $\mathcal{T F}_{\rho}(L)$ is Borel measurable as well. To see this, observe that $\mathcal{T F}_{\rho}(L)$ is equal to the closure in $k$ of the values obtained by evaluating countably many polynomials at $\operatorname{tr} \rho(l)$ and $\operatorname{tr} \rho(l)^{-1}$ where $l$ ranges over individual elements of $L$. Using the continuity of the map $S \rightarrow k, S \ni s \mapsto \operatorname{tr} \rho(s)$ we conclude that $\mathcal{T}$ is Borel measurable.

By assumption $\mathrm{J}_{G, S}(H)=\mathrm{I}_{S}(S)$ for $\mu$-almost every closed subgroup $H$. It follows that $\mu$-almost always $\operatorname{pr}_{S}(\Gamma \cap U)$ is Zariski-dense in $S$. Relying on Proposition 2.8 we deduce that the map $\mathcal{T}$ is invariant under the conjugation action of $G^{\dagger}$. Ergodicity implies that it must be essentially constant, as required.

Remark 5.5. Let $\rho$ be a $k$-representation and $\mu$ an ergodic invariant random subgroup of $G$. It follows that the trace field $\mathcal{T F}_{\rho}(H)$ is $\mu$-almost everywhere constant on $\operatorname{Sub}(G)$. However this elementary variant can be proved more directly.

Proof of Theorem 1.9. Let $k$ be a local field, $G$ a happy semisimple analytic group over $k$ without non-standard isogenies and $\mu$ an ergodic invariant random subgroup of $G$. We first deal with the statement involving the normal subgroup $N$ and discreteness. The Archimedean and non-Archimedean cases are treated separately.

If $k$ is Archimedean then by Proposition 5.3 there is a normal subgroup $N_{1} \triangleleft G$ so that ${\overline{H_{0}}}^{\mathrm{Z}}=N_{1}$ for $\mu$-almost every subgroup $H$ in $G$. There is an almost direct factor $R$ of $G$ so that $R_{0}=R^{+} \leq N_{1}$ and $N_{1} R / R$ is central (Proposition 2.3). Cartan's closed subgroup theorem [Ser09, LG 5.9] implies that $H_{0}$ is a Lie subgroup of $G$. Since ${\overline{H_{0}}}^{Z}=N_{1}$ we have that $\operatorname{Lie}\left(H_{0}\right)=\operatorname{Lie}\left(N_{1}\right)=\operatorname{Lie}\left(R_{0}\right)$ and $H_{0}=R_{0}$ for $\mu$-almost every $H$. We conclude the Archimedean case by taking $N=R_{0} \triangleleft G$.

If $k$ is non-Archimedean the proof is more involved. We reduce the situation to adjoint absolutely simple groups and rely on Pink's theorem, as follows.

Let $\bar{G}$ be the adjoint group of $G$. There is a central $k$-isogeny $G \stackrel{\bar{p}}{\rightarrow} \bar{G}$. Write $\bar{G}$ as a direct product of its $k$-simple factors $\bar{G}=\prod_{i \in I} \bar{G}_{i}$ over the finite set $I$. Denote $\bar{\mu}=\bar{p}_{*} \mu$ so that $\bar{\mu}$ is an ergodic invariant random subgroup of $\bar{p}(G)$.

It is well-known [Tit66, 3.1.2] that there are finite local field extensions $k_{i} / k$ and adjoint absolutely simple $k_{i}$-groups $S_{i}$ such that $\bar{G}_{i}$ is $k$-isomorphic to $\mathcal{R}_{k_{i} / k}\left(S_{i}\right)$ 
for every $i \in I$. Denote $S=\prod_{i \in I} S_{i}$. In particular $\bar{G}$ is isomoprhic to $\prod_{i \in I} S_{i}\left(k_{i}\right)$ as a topological group 8 . Let $\operatorname{pr}_{i}$ denote the projection $\bar{G} \rightarrow S_{i}\left(k_{i}\right)$.

We apply Proposition 5.3 with respect to the simple factor $S_{i}$ and the invariant random subgroup $\bar{\mu}$ for every $i \in I$. It implies that $\operatorname{pr}_{i}\left(H \cap K_{H}\right)$ is either Zariskidense or trivial in $S_{i}$ for $\bar{\mu}$-almost every closed subgroup $H$ and compact open subgroup $K_{H}$ in $\bar{G}$ depending on $H$. The index set $I$ is a disjoint union $I=I_{\mathrm{d}} \cup I_{\mathrm{t}}$ with $i \in I_{\mathrm{d}}$ or $i \in I_{\mathrm{t}}$ if $\operatorname{pr}_{i}\left(H \cap K_{H}\right)$ is essentially always Zariski-dense or trivial, respectively. In other words $i \in I_{\mathrm{t}}$ if and only if $\operatorname{pr}_{i}\left(H \cap K_{H}\right)$ is trivial for $\bar{\mu}$-almost every closed subgroup $H$ and some suitable compact open subgroup $K_{H}$.

Let $\rho_{i}$ be the $k$-representation of $S_{i}$ provided by Theorem 2.7. The trace field $\mathcal{T F}_{\rho_{i}}\left(\operatorname{pr}_{i}\left(H \cap K_{H}\right)\right)$ is $\bar{\mu}$-almost surely constant for every $i \in I_{\mathrm{d}}$ by Proposition 5.4 Let this trace field be denoted $l_{i}$ so that $l_{i} \subset k_{i}$ is a local field for $i \in I_{\mathrm{d}}$. The first part of Theorem 2.7 implies that the field extension $k_{i} / l_{i}$ is finite for $i \in I_{\mathrm{d}}$. For notational convenience denote $l_{i}=k_{i}$ for every $i \in I_{\mathrm{t}}$.

Let $\widetilde{S}_{i}$ denote the universal covering group of $S_{i}$. There is a central $k_{i}$-isogeny $\widetilde{s}_{i}: \widetilde{S}_{i} \rightarrow S_{i}$ and a generalized commutator map $[\cdot, \cdot]: S_{i} \times S_{i} \rightarrow \widetilde{S}_{i}$ for every $i \in I$. Denoting $\widetilde{S}=\prod_{i \in I} \widetilde{S}_{i}$ we obtain the maps $\widetilde{s}: \widetilde{S} \rightarrow S$ and $\widetilde{[\cdot, \cdot]}: S \times S \rightarrow \widetilde{S}$. The map $\widetilde{s}$ is continuous and proper on the rational points. The closure of the generalized commutator gives rise to a well-defined map gc : $\operatorname{Sub}(S) \rightarrow \operatorname{Sub}(\widetilde{S})$. Observe that the map gc is equivariant in the sense that

$$
g^{-1} \cdot \operatorname{gc}(H) \cdot g=\operatorname{gc}\left(\widetilde{s}(g)^{-1} \cdot H \cdot \widetilde{s}(g)\right),
$$

for all $g \in \widetilde{S}$ and $H \in \operatorname{Sub}(S)$. We take $\widetilde{\mu}=\operatorname{gc}_{*} \bar{\mu}$ so that $\widetilde{\mu}$ is an ergodic invariant random subgroup of $\widetilde{S}$.

Consider the semisimple analytic group $\widetilde{L}$ obtained by taking restriction of scalars

$$
\widetilde{L}=\prod_{i \in I} \mathcal{R}_{k_{i} / l_{i}}\left(\widetilde{S}_{i}\right)
$$

Denote $\widetilde{L}_{i}=\mathcal{R}_{k_{i} / l_{i}}\left(\widetilde{S}_{i}\right)\left(l_{i}\right)$ so that $\widetilde{S}_{i}$ is isomorphic to $\widetilde{L}_{i}$ as a topological group for every $i \in I$. Let $\widetilde{\mathrm{pr}}_{i}$ denote the projection from $\widetilde{S}$ to the factor $\widetilde{L}_{i}$. We argue once more relying on Proposition 5.3 , this time with respect to the factor $\widetilde{L}_{i}$ for $i \in I$ and the invariant random subgroup $\widetilde{\mu}$, deducing that $\widetilde{p r}_{i}\left(F \cap K_{F}\right)$ is either Zariski-dense or central in $\widetilde{L}_{i}$ for $\widetilde{\mu}$-almost every closed subgroup $F$ in $\widetilde{S}$ and a compact open subgroup $K_{F}$ depending on $F$. This depends on whether $\widetilde{p r}_{i}\left(F \cap K_{F}\right)$ is infinite or finite, respectively. Since the maps $\widetilde{s}_{i}$ are proper, the groups $\widetilde{p r}_{i}\left(F \cap K_{L}\right)$ are essentially always Zariski-dense or central depending on whether $i \in I_{\mathrm{d}}$ or $i \in I_{\mathrm{t}}$, respectively.

For every $i \in I_{\mathrm{d}}$ the groups $\widetilde{\operatorname{pr}}_{i}\left(F \cap K_{F}\right)$ are Zariski dense in $\mathcal{R}_{k_{i} / l_{i}}\left(\widetilde{S}_{i}\right) \widetilde{\mu}$-almost always. The second part of Theorem 2.7 implies that $\widetilde{p r}_{i}\left(F \cap K_{F}\right)$ is open in $\widetilde{S}_{i}$. On the other hand for every $i \in I_{\mathrm{t}}$, the groups $\widetilde{\operatorname{pr}}_{i}\left(F \cap K_{F}\right)$ are essentially always central. Since $K_{F}$ is allowed to be arbitrary small they are in fact trivial.

To draw conclusions denote $\widetilde{S}_{\mathrm{d}}=\prod_{i \in I_{\mathrm{d}}} \widetilde{S}_{i}$ and let $\widetilde{\mu}_{\mathrm{d}}$ be the ergodic invariant random subgroup of $\widetilde{S}_{\mathrm{d}}$ obtained from $\widetilde{\mu}$ by taking intersections with $\widetilde{S}_{\mathrm{d}}$ regarded as a closed subgroup of $\widetilde{S}$. The above discussion shows that $\widetilde{\mu}_{\mathrm{d}}$-almost every closed

\footnotetext{
${ }^{8}$ Making our customary abuse of notation, $S$ denotes an algebraic group as well as its group of rational points.
} 
subgroup $F \leq \widetilde{S}_{\mathrm{d}}$ admits an open projection to $\widetilde{S}_{i}$ for any $i \in I_{\mathrm{d}}$. However, a totally disconnected l.c.s.c. group has only countably many open subgroups. An ergodic probability measure on a countable set must be a point mass supported on a fixed point. Taking into account Proposition 2.3 we see that $\widetilde{\mu}$-almost every closed subgroup of $\widetilde{S}$ contains $\widetilde{S}_{\mathrm{d}}$.

We now translate these conclusions back to our groups of interest $\bar{G}$ and $G$. In particular $\bar{\mu}$-almost every closed subgroup $H$ of $\bar{p}(G)$ contains $S_{\mathrm{d}}^{+}$and $H S_{\mathrm{d}} / S_{\mathrm{d}}$ is discrete, where $S_{d}=\prod_{i \in I_{\mathrm{d}}} S_{i}$. Therefore $\mu$-almost every closed subgroup of $G$ contains $N=\bar{p}^{-1}\left(S_{\mathrm{d}}^{+}\right)$. Since $G$ is happy the quotient $G / G^{+}$is finite. This implies that $H N / N$ is discrete in $G / N$ for $\mu$-almost every subgroup $H$, as required.

Finally, it remains to deal with Zariski closure. The existence of a normal subgroup $M \triangleleft G$ so that $\bar{H}^{Z}=M$ for $\mu$-almost every closed subgroup $H$ follows immediately from the corresponding part of Proposition 5.3.

Whenever Lie algebra methods are available there is a simpler proof of the discreteness part avoiding Pink's theorem. This is the case for $k=\mathbb{R}$ and $k=\mathbb{Q}_{p}$, and this is the approach taken in $\left[\mathrm{ABB}^{+} 17\right]$ for real Lie groups.

\section{Semisimple analytic Groups ARE SElF-Chabauty-ISOlated}

We study of the following property for semisimple analytic groups.

Definition 6.1. A l.c.s.c. group $G$ is self-Chabauty-isolated if the point $G$ is isolated in $\operatorname{Sub}(G)$ with the Chabauty topology.

Note that $G$ is self-Chabauty-isolated if and only if there is a finite collection of open subsets $U_{1}, \ldots, U_{n} \subset G$ so that the only closed subgroup intersecting every $U_{i}$ non-trivially is $G$ itself. The main theorem in this section is the following.

Theorem 6.2. Let $G$ be a happy semisimple analytic group. Then $G^{+}$is selfChabauty-isolated.

Recall that in particular if $G$ is simply connected or has zero characteristic then it is happy. We will first consider the non-Archimedean case and then extend this to general semisimple analytic groups, allowing for connected factors.

6.1. The non-Archimedean case. Let $G$ be an happy non-Archimedean semisimple analytic group. The Chabauty-isolation of the subgroup $G^{+}$is easily deduced from the following three propositions.

Proposition 6.3. Let $H$ be a l.c.s.c. group and $O \leq H$ an open subgroup. Assume that there are only finitely many intermediate subgroups $O \leq Q \leq H$. If $O$ is selfChabauty-isolated then $H$ is self-Chabauty-isolated as well.

The above assumption holds in particular when $O$ is maximal or of finite index.

Proof. Let $Q_{1}, \ldots, Q_{n}$ be the distinct subgroups with $O \leq Q_{i} \lesseqgtr H$. Choose arbitrary elements $h_{i} \in H \backslash Q_{i}$ for every $i \in\{1, \ldots, n\}$. Since $O$ is self-Chabautyisolated, there are open subsets $U_{1}, \ldots, U_{m} \subset O$ so that the only closed subgroup of $O$ intersecting each $U_{j}$ non-trivially is $O$ itself. Then clearly

$$
\{H\}=\bigcap_{i=1}^{n} \mathcal{O}_{2}\left(U_{i}\right) \cap \bigcap_{i=1}^{m} \mathcal{O}_{2}\left(h_{i} O\right)
$$

and the latter subset is open in $\operatorname{Sub}(H)$, as required. 
Proposition 6.4. Let $P_{1}, \ldots, P_{n}$ be finitely generated pro- $p_{i}$ groups for some prime numbers $p_{i}$. Then the product $\prod_{i=1}^{n} P_{i}$ is a self-Chabauty-isolated group.

Proof. Follows immediately from GS10, 5.6].

Proposition 6.5. The group $G^{+}$has a self-Chabauty-isolated compact open subgroup.

If $G$ is $p$-adic then Proposition 6.5 follows from Lazard's theorem DDSMS03, Theorem 8.1]. Note that every non-Archimedean semisimple analytic group in zero characteristic can be regarded as a group defined over $\mathbb{Q}_{p}$ using restriction of scalars.

Proof. Let $S$ be a factor of $G$ so that $S$ is a simple analytic group over some local field $k$. If $k$ has zero characteristic let $p$ be such that $k$ is a finite extension of $\mathbb{Q}_{p}$. Otherwise let $p=\operatorname{char}(k)$. Taking into account Proposition 6.4 it will suffice to show that the group $S^{+}$admits a finitely generated pro- $p$ group.

Recall that $\mathcal{O}$ is the valuation ring and $\pi$ a uniformizer element of $k$. Assume that $S=\mathbb{S}(k)$ and $\mathbb{S} \leq \mathrm{GL}_{N}$ for some $N \in \mathbb{N}$. We claim that the first congruence subgroup $\mathbb{S}(\pi \mathcal{O})$ of $\mathbb{S}(\mathcal{O})$ is a pro- $p$ group 9 .

Consider the descending sequence of congruence subgroups $\mathbb{S}\left(\pi^{n} \mathcal{O}\right)$ for $n \in \mathbb{N}$. These form a basis of neighborhoods at the identity for $\mathbb{S}(\pi \mathcal{O})$. We need to verify that every successive quotient $P_{n}$ with $n \in \mathbb{N}$

$$
P_{n}=\mathbb{S}\left(\pi^{n} \mathcal{O}\right) / \mathbb{S}\left(\pi^{n+1} \mathcal{O}\right)
$$

is a $p$-group [RZ00, 2.3.2]. Let $s_{1}, s_{2} \in \mathbb{S}\left(\pi^{n} \mathcal{O}\right)$ be a pair of elements. We may write $s_{i}=\mathrm{Id}+\pi^{n} A_{i}$ for some matrices $A_{i} \in \mathrm{M}_{N}(\mathcal{O})$ and $i=1,2$. The product $s_{1} s_{2}$ is given by

$$
s_{1} s_{2}=\mathrm{Id}+\pi^{n}\left(A_{1}+A_{2}\right)+\pi^{n+1} \mathrm{M}_{N}(\mathcal{O})
$$

so that $s_{1} s_{2}=\operatorname{Id}+\pi^{n}\left(A_{1}+A_{2}\right)$ in $P_{n}$. This calculation shows that $P_{n}$ is an abelian group of exponent $p$ for every $n \in \mathbb{N}$, establishing the above claim.

Since $G$ is happy the Barnea-Larsen theorem, given here as Theorem 2.5, implies that the first congruence subgroup is finitely generated.

We are ready to show that $G^{+}$is self-Chabauty-isolated. The proof should be compared with [GM11, 3.3].

Theorem 6.6. Let $G$ be a happy non-Archimedean semisimple analytic group. Then the subgroup $G^{+}$is self-Chabauty-isolated.

Proof. Let $P$ be a self-Chabauty-isolated compact open subgroup of $G^{+}$provided by Proposition 6.5. Let $K$ be a maximal compact open subgroup so that $P \leq$ $K \leq G^{+}$. Clearly $P$ has finite index in $K$. According to Corollary 2.11 there are only finitely many intermediate subgroups between $K$ and $G^{+}$. The statement now follows by applying Proposition 6.4 twice with respect to the two pairs $P \leq K$ and $K \leq G^{+}$.

\footnotetext{
${ }^{9}$ See [PR92, Lemma 3.8] for a different proof of the fact that $\mathbb{S}(\pi \mathcal{O})$ is a pro- $p$ in the zero characteristic case.
} 
6.2. General semisimple analytic groups. We complete the proof of Chabauty isolation for general happy semisimple analytic group.

Proof of Theorem 6.2. Write $G^{+}$as an almost direct product $G^{+}=C \times D$ where $C$ and $D$ are connected and totally disconnected semisimple analytic groups, respectively. The two factors $C$ and $D$ are self-Chabauty-isolated by $\left[\mathrm{ABB}^{+} 17\right.$, Prop. 2.2] and Theorem 6.6. respectively. We are required to show that the almost direct product $G^{+}=C \times D$ is self-Chabauty-isolated as well.

Let $U_{1}, \ldots, U_{n} \subset C$ be open subsets so that the only closed subgroup of $C$ intersecting non-trivially each $U_{i}$ is $C$ itself. Similarly let $V_{1}, \ldots, V_{m} \subset D$ be open subsets satisfying the analogous property with respect to $D$.

By Proposition 6.5 there is a self-Chabauty-isolated compact open subgroup $K \leq D$. This implies that $K$ admits an open normal subgroup $P$ so that the only closed subgroup of $K$ intersecting non-trivially every coset of $P$ is $K$ itself. Let $k_{1}, \ldots, k_{t}$ be coset representatives for $P$ in $K$.

Consider the Chabauty-open subset $\Omega$ of $\operatorname{Sub}\left(G^{+}\right)$given by

$$
\Omega=\bigcap_{i=1}^{n} \mathcal{O}_{2}\left(U_{i} \times P\right) \cap \bigcap_{i=1}^{m} \mathcal{O}_{2}\left(C \times V_{j}\right) \cap \bigcap_{i=1}^{t} \mathcal{O}_{2}\left(C \times k_{i} P\right) .
$$

We complete the proof by showing that $\Omega=\left\{G^{+}\right\}$.

Let $L \in \Omega$ be any closed subgroup. Choose arbitrary elements $l_{i} \in L \cap\left(U_{i} \times P\right)$ for all $1 \leq i \leq n$ and denote $L^{\prime}=\left\langle l_{i}\right\rangle$. Observe that $L^{\prime} \leq C \times P$ and that $L^{\prime}$ projects densely to $C$. Next, choose elements $m_{i} \in L \cap\left(C \times k_{i} P\right)$ for $1 \leq i \leq t$. Note that

$$
e_{C} \in \overline{\operatorname{pr}_{C}\left(m_{i} L^{\prime}\right)} \text { and } \operatorname{pr}_{D}\left(m_{i} L^{\prime}\right) \subset k_{i} P .
$$

Since $L$ is closed and the cosets $k_{i} P$ are compact we deduce that

$$
L \cap\left(\left\{e_{C}\right\} \times k_{i} P\right) \neq \emptyset
$$

for every $1 \leq i \leq t$. Therefore $L$ contains the open subgroup $\left\{e_{C}\right\} \times K$.

The two projections of $L$ to $C$ and $D$ are dense by the definition of $\Omega$. In fact $L \cap(C \times P)$ already projects densely to $C$. The compactness of $P$ implies that $\operatorname{pr}_{C}(L)=C$. Since $L \cap\left(\left\{e_{C}\right\} \times D\right)$ is relatively open in $\left\{e_{C}\right\} \times D$ we have that $\operatorname{pr}_{D}(L)=D$.

We have established that $L$ surjects onto both factors $C$ and $D$. This implies that $L \cap\left(\left\{e_{C}\right\} \times D\right)$ is normal regarded as a subgroup of the factor $D$. The normal closure of $K$ in $D$ is the group $D$ itself. So $L$ contains $\left\{e_{C}\right\} \times D$. Since $L$ surjects onto the factor $C$ it follows that $L=C \times D=G^{+}$, as required.

Since $G$ is happy the quotient $G / G^{+}$is finite by Theorem 2.5. So that every subgroup $G^{\dagger}$ with $G^{+} \leq G^{\dagger} \leq G$ is clearly self-Chabauty-isolated as well.

Corollary 6.7 (Chabauty-isolation from discrete subgroups). Let $G$ be a happy semisimple analytic group, $N$ a non-discrete normal subgroup of $G$ and $H$ a closed subgroup of $G$ containing $N$ so that $H / N$ is discrete in $G / N$. Then $H$ cannot be approximated by discrete subgroups in $\operatorname{Sub}(G)$.

Proof. As was already observed above, the case where $G^{+} \leq N$ follows immediately from Theorem 6.2 combined with Proposition 6.3 and the finiteness of $G / G^{+}$. 
In all other cases we may according to Proposition 2.3 decompose $G$ into an almost direct product $G=G_{1} \times G_{2}$ of semisimple analytic groups such that $G_{1}^{+} \leq N$ and $H / G_{1}$ is discrete in $G / G_{1}$. The proof is by a few reduction steps.

If $G_{2}$ has a non-Archimedean semisimple analytic factor $G_{2, \text { td }}$ we may write $G$ as an almost direct product $G=G_{0} \times G_{2, \text { td }}$. Let $K$ be any compact open subgroup of $G_{2, \mathrm{td}}$. Note that the map

$$
\operatorname{Sub}(G) \rightarrow \operatorname{Sub}\left(G_{0}\right), \quad \Gamma \mapsto \operatorname{pr}_{G_{0}}\left(\Gamma \cap\left(G_{0} \times K\right)\right)
$$

is continuous and maps discrete subgroups in $G$ and to discrete subgroups in $G_{0}$, and likewise discrete subgroups in $G / N$ to discrete subgroups $G_{0} / N$. Therefore we may assume in what follows below that $G_{2}$ is Archimedean.

If $G_{1}$ is Archimedean as well then $G$ is a semisimple real Lie group. In this case it is a well known fact that the Chabauty limit of discrete groups has a nilpotent connected component and therefore cannot contain $\left(G_{1}\right)_{0}$, see e.g. $\left[\mathrm{ABB}^{+} 17,2.3\right]$.

If $G_{1}$ has a non-trivial Archimedean and a non-trivial non-Archimedean factors then $G_{1}$ splits into an almost direct product $G_{1}=G_{1, \mathrm{c}} \times G_{1, \mathrm{td}}$. We may combine the arguments of the two previous cases - namely, by intersecting with $G_{1, \mathrm{c}} \times K$ for a compact open subgroup $K \leq G_{1, \mathrm{td}}$, passing to the quotient $G_{1} / G_{1, \mathrm{td}}$ and then noting that discrete groups of $G / G_{1, \mathrm{td}}$ cannot have a subgroup containing $G_{1}^{+} / G_{1, t \mathrm{~d}}^{+}$as a limit point.

The remaining case is that $G_{1}$ is non-Archimedean and $G_{2}$ is Archimedean. Let $V$ be a relatively compact identity neighborhood in the real Lie group $G_{2}$ such that $V$ has no non-trivial closed subgroups and moreover $H \cap\left(G_{1} \times \bar{V}\right) \subset G_{1}$. Let $W$ be a symmetric identity neighborhood in $G_{2}$ satisfying $W^{2} \subset V$. By Proposition 6.5 the non-Archimedean group $G_{1}$ admits a self-Chabauty-isolated compact open subgroup $P$. Choose open subsets $U_{1}, \ldots, U_{n} \subset P$ so that every closed subgroup of $P$ that intersects each $U_{i}$ non-trivially must be $P$ itself. Consider the Chabautyopen set $\Omega$

$$
\Omega=\mathcal{O}_{1}(P \times(\bar{V} \backslash W)) \cap \bigcap_{j=1}^{n} \mathcal{O}_{2}\left(U_{j} \times W\right) .
$$

On the one hand clearly $H \in \Omega$. On the other hand, observe that every closed subgroup $L \in \Omega$ intersects non-trivially the subsets $U_{j} \times\left\{e_{G_{2}}\right\}$ for $j=1, \ldots, n$ and satisfies $L \cap(P \times V)=P \times\left\{e_{G_{2}}\right\}$. In particular, the Chabauty subspace of discrete subgroups in $G$ is disjoint from $\Omega$, as required.

As a special case of Corollary 6.7 we deduce that any non-discrete normal subgroup $N$ is Chabauty-isolated from the subspace of discrete subgroups in $G$.

\section{On Chabauty neighborhoods of Lattices}

We analyze the Chabauty neighborhoods of irreducible lattices in semisimple analytic groups. It turns out that in higher-rank there is a kind of local rigidity phenomenon, i.e. every irreducible lattice admits a Chabauty neighborhood consisting only of conjugates, see Theorem 1.10. We develop a general principle allowing to deduce this from the classical notion of local rigidity.

The first step is to show that Chabauty neighborhoods of lattices in semisimple analytic groups are jointly discrete in the following sense. 
Definition 7.1. A subgroup $\Delta \leq H$ admits a jointly discrete Chabauty neighborhood if there exist an identity neighborhood $V$ in $H$ and a Chabauty open neighborhood $\Omega$ of $\Delta$ in $\operatorname{Sub}(H)$ so that every closed subgroup $L \in \Omega$ satisfies $L \cap V=\{e\}$.

Theorem 7.2. Every lattice $\Gamma$ in a semisimple analytic group $G$ admits a jointly discrete Chabauty neighborhood.

Note that the lattice $\Gamma$ is not assumed to be irreducible. In the following two subsections we deal with the non-Archimedean case of Theorem 7.2

7.1. Strongly regular hyperbolic elements. Let $G$ be a non-Archimedean semisimple analytic group and $X$ the associated Bruhat-Tits building.

Definition 7.3. An element $\gamma \in G$ is strongly regular hyperbolic if $\gamma$ is hyperbolic and the two endpoints of any translation axis for $\gamma$ lie in the interiors of two opposite chambers of the spherical building at infinity.

The notion of strongly regular elements was introduced by Caprace and Ciobotaru [CC15. This is a generalization of the notion of $\mathbb{R}$-hyper-regular elements in semisimple real Lie groups [PR72].

The minimal set10 of a strongly regular element of $G$ is a uniquely determined apartment in the building $X$ CC15, 2.3]. In particular, a strongly regular hyperbolic element belongs to a unique maximal $k$-split torus.

Proposition 7.4. Let $\gamma \in G$ be a strongly regular hyperbolic element. Then there is an open identity neighborhood $U$ in $G$ and a number $N_{\gamma} \in \mathbb{N}$ so that for $n \geq N_{\gamma}$ every element $\gamma^{\prime} \in U \gamma^{n} U$ is strongly regular hyperbolic and satisfies $\operatorname{Min}\left(\gamma^{\prime}\right)=$ $u \operatorname{Min}(\gamma)$ for some $u \in U$.

Proof. Let $\Delta$ be the unique apartment in the building $X$ with $\operatorname{Min}(\gamma)=\Delta$. Let $l: \mathbb{R} \rightarrow X$ be a translation axis for $\gamma$ and assume that $x_{0}=l(0)$ is a special vertex. So $l(\mathbb{R})$ is a geodesic line contained in the apartment $\Delta$ and crossing every wall11 CC15, 2.2]. We have $\gamma l(t)=l(t+d)$ for some $d>0$ and all $t \in \mathbb{R}$.

Choose $N_{\gamma} \in \mathbb{N}$ sufficiently large so that the two special vertices $x_{0}$ and $\gamma^{n} x_{0}$ belong to the interior of opposite sectors 12 of $\Delta$ for all $n \geq N_{\gamma}$.

Denote $I=[0,1]$ and let $U \leq G$ be a compact open subgroup fixing the set $l(I)$ point-wise. Consider an element $\gamma^{\prime} \in G$ of the form

$$
\gamma^{\prime}=u_{1} \gamma^{n} u_{2}, \quad u_{1}, u_{2} \in U, n \geq N_{\gamma} .
$$

Denote $l_{1}=u_{1} \circ l$ so that $l_{1}(\mathbb{R})$ is a geodesic line contained in the apartment $\Delta_{1}=u_{1} \Delta$ satisfying $l(t)=l_{1}(t)$ for all $t \in I$. Therefore

$$
\gamma^{\prime} l_{1}(t)=u_{1} \gamma^{n} u_{2} l_{1}(t)=u_{1} \gamma^{n} l(t)=u_{1} l(t+n d)=l_{1}(t+n d)
$$

for all $t \in I$.

This calculation implies that $\gamma^{\prime}$ is a hyperbolic element admitting some translation axis $l^{\prime}: \mathbb{R} \rightarrow X$ containing the geodesic segment $l_{1}([0, n d])$ [CC15, 2.8]. The

\footnotetext{
${ }^{10}$ The minimal set $\operatorname{Min}(\gamma)$ of the element $\gamma$ is the set of points on which the minimal displacement of $\gamma$ is attained. $\operatorname{Min}(\gamma)$ is a closed convex subset of $X$. See [BH11 II.6].

${ }^{11} \mathrm{~A}$ wall in $\Delta$ is the fixed point set of a reflection by an element in the affine Weyl group Gar97 p. 34].

${ }^{12} \mathrm{~A}$ sector in $\Delta$ at the vertex $x_{0}$ is a connected component of the complement of all walls passing through $x_{0}$. It is a simplicial cone $x_{0}+C$. The two sectors $x_{0}+C$ and $x_{0}-C$ are opposite Gar97, p. 221]
} 
two special vertices $x_{0}$ and $\gamma^{\prime} x_{0}=u_{1} \gamma^{n} x_{0}=l^{\prime}(n d)=l_{1}(n d)$ lie along the translation axis $l^{\prime}$ and belong to opposite sectors of the apartment $\Delta_{1}$. Relying on the local criterion of [CC15, 2.5] we deduce that $\gamma^{\prime}$ is strongly regular hyperbolic.

The minimal set $\operatorname{Min}\left(\gamma^{\prime}\right)$ is equal to the unique apartment $\Delta^{\prime}$ containing the axis $l^{\prime}$. By the strong transitivity property of buildings there is an element $u \in G$ satisfying

$$
u \Delta=\Delta^{\prime} \quad \text { and } \quad u_{\mid \Delta \cap \Delta^{\prime}}=\mathrm{id} .
$$

The geodesic segment $l(I)=l_{1}(I)=l^{\prime}(I)$ is contained in $\Delta \cap \Delta^{\prime}$ and therefore $u \in U$. The proof is complete as $\Delta=\operatorname{Min}(\gamma)$ and $\Delta^{\prime}=\operatorname{Min}\left(\gamma^{\prime}\right)$.

Proposition 7.5. Every lattice $\Gamma$ in $G$ contains strongly regular hyperbolic elements.

Proof. The group $G$ contains strongly regular hyperbolic elements according to Theorem 1.2 of [CC15]. It is now standard to deduce that $\Gamma$ has strongly regular hyperbolic elements as well, relying on Proposition 7.4. In fact, we only need to use the fact that $\Gamma$ has the so called property (S) $\operatorname{Rag} 76,5.1,5.4$.

7.2. Joint discreteness in the non-Achimedean case. We are ready to prove the following non-Archimedean case of Theorem [7.2. Recall that jointly discrete Cahabauty neighborhoods were introduced in Definition 7.1 above.

Theorem 7.6. Every lattice $\Gamma$ in a non-Archimedean semisimple analytic group $G$ admits a jointly discrete Chabauty neighborhood.

We will make use of the following lemma.

Lemma 7.7. Let $S$ be a maximal $k$-split torus and $\Gamma$ any Zariski-dense subgroup in $G$. Then there are elements $\gamma_{1}, \ldots, \gamma_{m} \in \Gamma$ for some $m \in \mathbb{N}$ and an identity neighborhood $W \subset G$ so that the intersection $\bigcap_{i=1}^{m} S^{\gamma_{i} w_{i}}$ is central in $G$ for every choice of elements $w_{i} \in W$.

Proof. Consider the intersection $S_{0}=\bigcap_{\gamma \in \Gamma} S^{\gamma}$ as well as the group $\tilde{S}=\left\langle S^{\gamma}: \gamma \in\right.$ $\Gamma\rangle$. Since $\Gamma$ is Zariski dense, the Zariski closure of $\tilde{S}$ is normal in $G$, and as it contains a maximal split torus it must be all of $G$. Since $S_{0}$ is central in $\tilde{S}$, it is also central in $G$. The conclusion now follows from Proposition 4.5

Proof of Theorem 7.6. Let $G$ be a non-Archimedean semisimple analytic group, $X$ the corresponding Bruht-Tits building and $\Gamma$ a lattice in $G$.

The lattice $\Gamma$ contains a strongly regular hyperbolic element $\gamma$ by Proposition 7.5. The element $\gamma$ preserves the apartment $\Delta=\operatorname{Min}(\gamma)$ in $X$. Let $S$ be the unique maximal $k$-split torus in $G$ containing $\gamma$. Note that $S$ preserves $\Delta$ as well.

Making use of Lemma 7.7 we find elements $\gamma_{1}, \ldots, \gamma_{m} \in \Gamma$ for some $m \in \mathbb{N}$ and an identity neighborhood $W \subset G$ such that $\bigcap_{i=1}^{m} S^{\gamma_{i}^{\prime}}$ is central in $G$ for all $\gamma_{i}^{\prime} \in \gamma_{i} W$. Every conjugate $\gamma^{\gamma_{i}}=\gamma_{i}^{-1} \gamma \gamma_{i}$ is strongly regular hyperbolic as well. Let $U$ be an identity neighborhood contained in $W$ and $N \in \mathbb{N}$ be such that every element $\lambda_{i} \in U\left(\gamma^{N}\right)^{\gamma_{i}} U$ is strongly regular hyperbolic, as in Proposition 7.4. The unique $k$-split torus containing $\left(\gamma^{N}\right)^{\gamma_{i}}$ is still $S_{i}^{\gamma}$ and the second part of Proposition 7.4 shows that $\lambda_{i} \in S^{\gamma_{i} u_{i}}$ for some $u_{i} \in U \subset W$.

Fix an additional relatively compact identity neighborhood $V$ in $G$. Relying on Lemma 9.4 of GL16] we may construct a Chabauty neighborhood $\Omega$ of $\Gamma$ in $\operatorname{Sub}(G)$ with the following properties - every closed subgroup $L \in \Omega$ satisfies that 
- $L_{V}=L \cap V$ is a subgroup of $G$,

- $L$ contains elements $\lambda_{i} \in U\left(\gamma^{N}\right)^{\gamma_{i}} U$ for all $i=1, \ldots, m$,

- the subgroup $L_{V}$ is normalized by $L_{0}=\left\langle\lambda_{1}, \ldots, \lambda_{m}\right\rangle \leq L$, and

- $L_{V} \cap Z(G)=\{e\}$ where $Z(G)$ is the center of $G$.

The last of the above requirements does not appear in [GL16]. However $Z(G)$ is finite and it is clear that we may add this additional requirement on $\Omega$.

We claim that for every closed subgroup $L \in \Omega$ the intersection $L_{V}=L \cap V$ consists of the identity element. Consider some $L \in \Omega$ and let $F \subset X$ denote the closed convex subset of $L_{V}$-fixed points. The compactness of the subgroup $L_{V}$ implies that $F$ is nonempty. Clearly $F$ is $L_{0}$-invariant.

By construction the elements $\lambda_{i}$ are strongly regular hyperbolic. Since $F$ is $\lambda_{i}$ invariant its boundary $\partial F$ contains the two endpoints $l_{i}(\infty)$ and $l_{i}(-\infty)$ of some translation axis $l_{i}$ for $\lambda_{i}$. In particular $\partial F$ is nonempty. Since $L_{V}$ fixes $F$ it must also fix the two endpoints $l_{i}(\infty)$ and $l_{i}(-\infty)$. These endpoints belong to the interiors of opposite chambers at infinity and $L_{V}$ must preserve the unique apartment determined by this pair. Therefore $L_{V}$ is contained in the torus $S^{\gamma_{i} u_{i}}$. To conclude

$$
L_{V} \leq L_{V} \cap \bigcap_{i=1}^{m} S^{\gamma_{i} u_{i}} \leq L_{V} \cap Z(G)=\{e\} .
$$

We have shown that $\Gamma$ admits a jointly discrete Chabauty neighborhood $\Omega$.

7.3. Joint discreteness in the general case. We extend Chabauty joint discreteness to general semisimple analytic groups, making use of the fact that Lie groups have no small subgroups.

Proof of Theorem 7.2. Write $G$ as an almost direct product $G=C \times D$ where $C$ and $D$ are connected and totally disconnected semisimple analytic groups, respectively. Let $\Gamma$ be a lattice in $G$. The proof of Theorem 7.6 given above can be easily adapted to this case, as follows.

Let $V_{C} \subset C$ be an identity neighborhood which does not contain any closed non-trivial subgroup. Let $V_{D} \leq D$ be a relatively compact identity neighborhood as in the proof of Theorem 7.6. Take $V=V_{C} \times V_{D}$ to be used in the definition of the Chabauty neighborhood $\Omega$. Note that every closed subgroup $L \in \Omega$ satisfies $\overline{\operatorname{pr}_{C}(L \cap V)}=\left\{e_{C}\right\}$, so that $L_{V}=L \cap V$ can be regarded as a subgroup of the non-Archimedean factor $D$.

The argument of Proposition 7.5 implies that $\Gamma$ admits an element $\gamma$ whose projection to $D$ is strongly regular hyperbolic. The rest of the proof of Theorem 7.6 applies with no changes.

7.4. Local rigidity and Chabauty neighborhoods. Let $G$ be a topological group and $\Gamma \leq G$ a lattice. Let $\operatorname{Hom}(\Gamma, G)$ denote the space of all homomorphisms from $\Gamma$ to $G$ with the point-wise convergence topology. If $\Sigma$ is a generating set for $\Gamma$ then $\operatorname{Hom}(\Gamma, G)$ can be identified with a closed subset of $G^{\Sigma}$. The lattice $\Gamma$ is weakly locally rigid if the inclusion morphism $\iota: \Gamma \rightarrow G$ admits an open neighborhood in $\operatorname{Hom}(\Gamma, G)$ consisting of conjugates by an automorphism of $G$. $\Gamma$ is locally rigid if this automorphism is inner [GL16, Sec. 2].

Definition 7.8 (Relative finite presentability). $\Gamma$ is finitely presented inside $G$ if it has a finite generating set $\Sigma$ and there are finitely many words $\omega_{1}, \ldots, \omega_{n} \in F_{\Sigma}$ 
so that

$$
\operatorname{Hom}(\Gamma, G)=\left\{\left(g_{\sigma}\right) \in G^{\Sigma}: \omega_{i}\left(g_{\sigma}\right)=e_{G} \forall i \in\{1, \ldots, n\}\right\}
$$

Here $F_{\Sigma}$ in the free group on the generators $\Sigma$.

This notion is clearly independent of the choice of a particular finite generating set for $\Gamma$. If $\Gamma$ itself is finitely presented then it is finitely presented inside any group. Moreover any finitely generated group is finitely presented inside an algebraic group by Noetherianity.

We are ready to formulate a general principle allowing to promote classical local rigidity to its the Chabauty topology variant.

Proposition 7.9. Let $G$ be a locally compact group whose automorphism group preserves the Haar measure. Let $\Gamma$ be a weakly locally rigid lattice in $G$ which is finitely presented inside $G$ and admits a jointly discrete Chabauty neighborhood.

Then $\Gamma$ has a Chabauty neighborhood consisting of conjugates by an automorphism of $G$. If $\Gamma$ is moreover locally rigid then this automorphism is inner.

Proof. Let $\Sigma$ be a finite generating set for $\Gamma$ and $\omega_{1}, \ldots, \omega_{n} \in F_{\Sigma}$ word 13 exhibiting $\Gamma$ to be finitely presented inside $G$ as in Definition 7.8 . Let $m=\max _{i=1, \ldots, n}\left|\omega_{i}\right|$ denote the maximal length of the words $\omega_{i}$.

Since $\Gamma$ is weakly locally rigid there is an identity neighborhood $U$ in $G$ so that every group homomorphism $f: \Gamma \rightarrow G$ with $f(\sigma) \in U \sigma$ for every $\sigma \in \Sigma$ is given by

$$
f(\gamma)=\alpha(\gamma), \quad \forall \gamma \in \Gamma
$$

for some automorphism $\alpha$ of $G$. If $\Gamma$ is assumed to be locally rigid then $\alpha$ is inner.

Joint discreteness allows us to choose an identity neighborhood $V$ in $G$ and an open Chabauty neighborhood $\Omega_{1} \subset \operatorname{Sub}(G)$ of $\Gamma$ so that every closed subgroup $L \in$ $\Omega_{1}$ satisfies $L \cap V=\{e\}$. Denote $\Sigma_{0}=\Sigma \cup\{e\}$ and take an identity neighborhood $W$ in $G$ satisfying

$$
W \subset U \text { and }\left(W^{\left(\Sigma_{0}^{m-1}\right)}\right)^{m} \subset V .
$$

Let $\Omega_{2} \subset \operatorname{Sub}(G)$ be a Chabauty neighborhood of $\Gamma$ so that every closed subgroup $L \in \Omega_{2}$ contains elements $l_{\sigma} \in W \sigma$ for every $\sigma \in \Sigma$. The argument of Rag76, p. 28] shows that there is a Chabauty neighborhood $\Omega_{3} \subset \operatorname{Sub}(G)$ of $\Gamma$ so that every $L \in \Omega_{1} \cap \Omega_{3}$ satisfies $\operatorname{co-vol}(L)>\frac{1}{2} \operatorname{co}-\operatorname{vol}(\Gamma)$.

Consider a closed subgroup $L \leq G$ with $L \in \Omega_{1} \cap \Omega_{2} \cap \Omega_{3}$. There are elements $l_{\sigma} \in L \cap W \sigma$ for $\sigma \in \Sigma$ as above. We claim that the homomorphism $\varphi$ given by

$$
\varphi: F_{\Sigma} \rightarrow L, \quad \varphi(\sigma)=l_{\sigma} \quad \forall \sigma \in \Sigma
$$

represents an element of $\operatorname{Hom}(\Gamma, G)$. Write $\omega_{i}=\sigma_{1} \cdots \sigma_{m_{i}}$ with $\sigma_{j} \in \Sigma$. Then

$$
\varphi\left(\omega_{i}\right) \in W \sigma_{1} W \sigma_{2} W \cdots W \sigma_{m_{i}} \subset W W^{\sigma_{1}} W^{\sigma_{1} \sigma_{2}} \cdots W^{\sigma_{1} \cdots \sigma_{m_{i}-1}} \omega_{i} .
$$

However the word $\omega_{i}=\sigma_{1} \cdots \sigma_{m_{i}}$ evaluated in $G$ is equal to the identity. Therefore

$$
\varphi\left(\omega_{i}\right) \in L \cap\left(W^{\left(\Sigma_{0}\right)^{m-1}}\right)^{m} \subset L \cap V=\left\{e_{G}\right\}
$$

Since $\Gamma$ is presented inside $G$ by $\left\{\omega_{i}, i=1, \ldots, n\right\}$ we obtain a well-defined homomorphism $\psi \in \operatorname{Hom}(\Gamma, G)$ by taking

$$
\psi: \Gamma \rightarrow G, \quad \psi(\sigma)=l_{\sigma} \quad \forall \sigma \in \Sigma
$$

\footnotetext{
${ }^{13}$ If $G$ is an algebraic group then these words $\omega_{i}$ define $\operatorname{Hom}(\Gamma, G)$ as an algebraic variety.
} 
By the above construction and as $\Gamma$ is weakly locally rigid there is an automorphism $\alpha$ of $G$ so that $\psi(\gamma)=\alpha(\gamma)$ for all $\gamma \in \Gamma$. The group $L$ is discrete and contains $\alpha(\Gamma)$ so that $L$ is a lattice. By assumption the automorphism $\alpha$ preserves Haar measure so that $\operatorname{co-} \operatorname{vol}(\alpha(\Gamma))=\operatorname{co}-\operatorname{vol}(\Gamma)$. We obtain

$$
2>\operatorname{co-vol}(\Gamma) / \operatorname{co-} \operatorname{vol}(L)=\operatorname{co-} \operatorname{vol}(\alpha(\Gamma)) / \operatorname{co}-\operatorname{vol}(L)=[L: \psi(\Gamma)] \in \mathbb{N} .
$$

This can only be the case provided that $\psi(\Gamma)=\alpha(\Gamma)=L$, as required.

If $\Gamma$ is moreover assumed to be locally rigid then $\alpha$ is inner. In that case $\alpha$ clearly preserves the Haar measure on $G$ and Proposition 2.13 becomes redundant.

7.5. Chabauty Local rigidity in semisimple analytic groups. We complete the proof that an irreducible lattice in a higher rank semisimple analytic group admits a Chabauty neighborhood consisting of conjugates.

Proof of Theorem 1.10. Let $G$ be a semisimple analytic group with $\operatorname{rank}(G) \geq 2$ and $\Gamma \leq G$ an irreducible lattice. The conclusion of Theorem 1.10 will follow from Proposition 7.9 as soon as we verify all of the required assumptions.

The automorphism group of $G$ preserves Haar measure by Proposition 2.13 . The lattice $\Gamma$ is finitely generated [Mar91, IX.3] and finitely presented inside $G$ in the sense of Definition 7.8 by Noetherianity. The fact that $\Gamma$ admits a jointly discrete Chabauty neighborhood is established in Theorem 7.2 .

It follows from Margulis super-rigidity theorem that $\Gamma$ is weakly locally rigid, and that $\Gamma$ is locally rigid provided that $\operatorname{char}(k)=0$ [Mar91, p. 241, Thms. A,C].

7.6. Wang's finiteness in the non-Archimedean case. We deduce Wang's finiteness relying on Chabauty local rigidity. This is essentially a standard argument appearing already in Wan72.

Proof of Theorem 1.12. Let $G$ be a semisimple analytic group with $\operatorname{rank}(G) \geq 2$. We may assume that all of the simple factors of $G$ are of the same characteristic, for otherwise $G$ admits no irreducible lattices.

We claim that $G$ has the Kazhdan-Margulis property for lattices [KM68, Gel15, GL16. This means that there is an identity neighborhood $U$ in $G$ such that every lattice $\Gamma$ in $G$ admits a conjugate intersecting $U$ only at the identity. If $G$ is Archimedean then this is a classical result — see [KM68] and also Gel15. If $G$ is zero characteristic non-Archimedean then much more is true, namely all lattices in $G$ are jointly discrete Ser09, LG 4.27, Th. 5]. If $G$ is simply-connected and positive characteristic non-Archimedean then $G$ has the Kazhdan-Margulis property by Theorem A of Gol09]. In the remaining case $G=C \times D$ where $C$ is Archimedean and $D$ is zero characteristic non-Archimedean semisimple analytic groups. Let $K \leq D$ be any compact open subgroup. For any lattice $\Gamma$ in $G$ the projection of $\Gamma \cap(C \times K)$ to $C$ is a lattice. Therefore if $U$ is a Kazhdan-Margulis neighborhood in $C$ then so is $U \times K$ in $G$.

Fix a co-volume $v>0$. Let $\Omega_{v}$ denote the Chabauty subspace of $\operatorname{Sub}(G)$ consisting of lattices of co-volume bounded above by $v$ and intersectiong the KazhdanMargulis neighborhood of $G$ only at the identity. The Chabauty criterion Rag76, 1.20] implies that $\Omega_{v}$ is compact. We conclude the proof of Wang's finiteness relying on Chabauty local rigidity as in Theorem 1.10 . 


\section{ACCUMUlation POINTS OF INVARIANT RANDOM SUBGROUPS}

We prove our main result on accumulation points of lattices in higher rank semisimple analytic groups with property $(T)$, generalizing the analog result of $\mathrm{ABB}^{+} 17$ from the classical Archimedean case to the general one.

Just as in $\mathrm{ABB}^{+} 17$ the proof relies on the celebrated rigidity theorem of Stuck and Zimmer SZ94. This theorem essentially provides classifies the ergodic invariant random subgroups in the situation under consideration. In our general setup we shall also require the non-Archimedean version of this theorem which was established in [Lev17, as well as the results obtained in $\$ 6$ and $\$ 7$.

Proof of Theorem 1.1. Let $G$ be an happy semisimple analytic group with $\operatorname{rank}(G) \geq$ 2 and property $(T)$. Let $\Gamma_{n}$ be a sequence of irreducible lattices in $G$ and assume that the $\Gamma_{n}$ 's are pairwise non-conjugate in the appropriate sense. By this we mean that the $\Gamma_{n}$ 's are pairwise non-conjugate in zero characteristic and pairwise non-conjugate by an automorphism of $G$ in positive characteristic 14 .

Let $\mu_{n}$ denote the invariant random subgroup corresponding to the lattice $\Gamma_{n}$ and consider a weak-* accumulation point $\mu \in \operatorname{IRS}(G)$ of the sequence $\mu_{n}$. The proof consists in showing that $\mu$ must be $\delta_{Z}$ for some central subgroup $Z$ in $G$. Our strategy is to proceed by elimination.

Since every factor of the group $G$ has property $(T)$ the accumulation point $\mu$ is irreducible by a result of Glasner-Weiss GW97. The Stuck-Zimmer rigidity theorem SZ94, Lev17] applies in this situation. This means that the $G$-action on $(\operatorname{Sub}(G), \mu)$ is either essentially transitive or has central stabilizers. The stabilizer of each point $H \in \operatorname{Sub}(G)$ is the normalizer $N_{G}(H)$ hence the second possibility is ruled out.

We may assume that $\mu$-almost every subgroup is conjugate to some fixed closed subgroup $H \leq G$. In particular $(\operatorname{Sub}(G), \mu$ ) is isomorphic as a $G$-space to the homogeneous space $G / N_{G}(H)$. This homogeneous space supports a $G$-invariant probability measure. The main result of [Mar77] therefore implies that

$$
N_{G}(H)=M \times \Gamma
$$

so that $G$ can be written as an almost direct product $G=G_{1} \times G_{2}$ with $G_{1}^{+} \leq M \leq$ $G_{1}$ and $\Gamma$ being a lattice in $G_{2}$. Examining the various possibilities for $H$ shows that it must be of the form $H=N \times \Delta$ where $N \triangleleft M$ and $\Delta \triangleleft \Gamma$ is a normal subgroup with $N_{G_{2}}(\Delta)=\Gamma$.

We claim that $G_{1}$ must be either trivial or equal to $G$. For if $G_{1}$ is a non-trivial proper subgroup of $G$ then $G_{1}^{+}$lies in the kernel of the $G$-action on the non-atomic homogeneous space $G /(M \times \Gamma)$ which is a contradiction to irreducibility.

If $G_{1}$ is trivial then $\Gamma$ is an irreducible lattice in $G$ and $\Delta$ is a normal subgroup of $\Gamma$. The normal subgroup theorem of Margulis [Mar91, Chapter IV] implies that $\Delta$ is either a lattice or central in $G$. In the current situation $\Delta$ must indeed be an irreducible lattice since $N_{G}(\Delta)=\Gamma$. However $\mu_{\Delta}$ is an isolated point in the extreme points of IRS $(G)$ by Corollary 1.11 and we arrive at a contradiction.

If $G_{1}$ is equal to $G$ then $H=N \triangleleft G$ is a normal subgroup. Since $\mu$ is a limit of invariant random subgroups supported on discrete subgroups, Corollary 6.7 implies that $H$ must be discrete and in particular central in $G$.

\footnotetext{
${ }^{14}$ In view of the Borel-Prasad finiteness theorem [BP89] we have in our situation that $\operatorname{vol}\left(G / \Gamma_{n}\right) \rightarrow \infty$. This fact however is not needed for the proof.
} 


\section{Limit FORMUla FOR NORMALIZED BetTi Numbers}

We prove a limit formula for the normalized Betti numbers as in Theorem 1.7 Note that the next and final section $\$ 10$ contains a much more general result concerning the limit of normalized relative Plancherel measures in the zero characteristic case. The argument of current section however is straightforward relying on a beautiful result of Elek and Lück's approximation theorem [Lüc94], and it applies in arbitrary characteristic.

Let $b_{d}(\Sigma)$ denote the $d$-th Betti number of a given simplicial complex $\Sigma$. We make use of the following general result due to Elek [Ele10, Lemma 6.1].

Theorem 9.1 (Elek). Let $\Sigma_{i}$ be a Benjamini-Schramm convergent sequence of finite simplicial complexes with uniformly bounded degree. Then the limit of $\frac{b_{d}\left(\Sigma_{i}\right)}{\left|V\left(\Sigma_{i}\right)\right|}$ exists as $i \rightarrow \infty$ for every $d \in \mathbb{N} \cup\{0\}$.

Note that a specific Benjamini-Schramm limit is not assumed for the sequence $\Sigma_{i}$ and that the limit of the normalized Betti numbers is not specified.

Proof of Theorem 1.7. Let $G$ be a non-Archimedean semisimple analytic group and $X$ the associated Bruhat-Tits building. Let $\Gamma_{i}$ be a sequence of torsion-free uniform lattices in $G$ so that the corresponding invariant random subgroups $\mu_{\Gamma_{i}}$ weak-* converge to $\delta_{\{e\}}$.

Let $\Delta \leq G$ be a fixed arbitrary uniform torsion-free lattice. The lattice $\Delta$ is residually finite. Therefore $\Delta$ has a descending sequence of normal subgroups $\Delta_{i} \triangleleft \Delta$ with $\bigcap_{i \in \mathbb{N}} \Delta_{i}=\{e\}$. By Lück's approximation theorem

$$
b_{d}^{(2)}(\Delta)=\lim _{i \rightarrow \infty} \frac{b_{d}\left(\Delta_{i}\right)}{\left[\Delta: \Delta_{i}\right]} \quad \forall d \in \mathbb{N} \cup\{0\} .
$$

Consider a sequence of lattices alternating between the $\Gamma_{i}$ 's at the odd positions and $\Delta_{i}$ 's at the even positions. The assumption on the $\Gamma_{i}$ 's together with Example 3.6 imply that the associated invariant random subgroups converge to $\delta_{\{e\}}$. The associated alternating sequence $\Sigma_{i}$ of simplicial complexes is given by

$$
\Sigma_{i}= \begin{cases}\Gamma_{j} \backslash X, & i=2 j-1 \\ \Delta_{j} \backslash X, & i=2 j\end{cases}
$$

Observe that $\Sigma_{i}$ is a convergent sequence in the Benjamini-Schramm sense by Corollary 3.11. Elek's theorem implies that the limit of $\frac{b_{d}\left(\Sigma_{i}\right)}{\left|V\left(\Sigma_{i}\right)\right|}$ exists for every $d \in \mathbb{N} \cup\{0\}$ and as $i \rightarrow \infty$. Restricting to the even positions we get

$$
\lim _{j \rightarrow \infty} \frac{b_{d}\left(\Sigma_{2 j}\right)}{\left|V\left(\Sigma_{2 j}\right)\right|}=\frac{1}{|V(X / \Delta)|} \lim _{j \rightarrow \infty} \frac{b_{d}\left(\Delta_{j}\right)}{\left[\Delta: \Delta_{j}\right]}=\frac{\mu_{G}(K)}{\kappa_{G}} \frac{b_{d}^{(2)}(\Delta)}{\operatorname{vol}(G / \Delta)}, \quad \forall d \in \mathbb{N} \cup\{0\} .
$$

Here $\mu_{G}$ is the Haar measure on $G, K$ is a maximal compact subgroup in $G$ and $\kappa_{G}$ is the number of vertices in a fundamental domain for the $G$-action on $X$.

The limit of the normalized Betti numbers is the same along the odd subsequence $\Sigma_{2 j-1}$ associated to the lattices $\Gamma_{j}$. Therefore

$$
\lim _{j \rightarrow \infty} \frac{b_{d}\left(\Gamma_{j}\right)}{\operatorname{vol}\left(G / \Gamma_{j}\right)}=\frac{\kappa_{G}}{\mu_{G}(K)} \lim _{j \rightarrow \infty} \frac{b_{d}\left(\Sigma_{2 j-1}\right)}{\left|V\left(\Sigma_{2 j-1}\right)\right|}=\frac{b_{d}^{(2)}(\Delta)}{\operatorname{vol}(G / \Delta)}, \quad \forall d \in \mathbb{N} \cup\{0\} .
$$

The right hand side is equal to $b_{d}^{(2)}(G)$ for every $d \in \mathbb{N} \cup\{0\}$, as required. 


\section{Convergence of Relative Plancherel measures}

Let $G$ be a semisimple analytic group over zero characteristic local fields. We prove Theorem 1.3 about convergence of normalized relative Plancherel measures.

10.1. Plancherel measure and the Sauvageot principle. Let $\widehat{G}$ denote the unitary dual of $G$, i.e. the set of all equivalence classes of unitary irreducible representations of $G$. The unitary dual $\widehat{G}$ is a topological space with the Fell topology. The Plancherel measure $\nu^{G}$ on $\widehat{G}$ is associated to the right regular representation of $G$ in $L^{2}\left(G, \mu_{G}\right)$. Note that $\nu^{G}$ depends the choice of Haar measure up to scaling.

Let $\varphi \in C_{c}^{\infty}(G)$ be a smooth function 15 with compact support. For every irreducible representation $\pi \in \widehat{G}$ the operator $\pi(\varphi)$ on $L^{2}\left(G, \mu_{G}\right)$ is trace class. The Fourier transform $\widehat{\varphi}$ is the function on $\widehat{G}$ given by

$$
\widehat{\varphi}: \pi \mapsto \operatorname{trace} \pi(\varphi)
$$

Theorem 10.1 (Plancherel formula). $\nu^{G}(\widehat{\varphi})=\varphi(e)$ for every $\varphi \in C_{c}^{\infty}(G)$.

Proof. This is the Fourier inversion formula, being a consequence of the Plancherel formula. It holds true for unimodular l.c.s.c. groups of type I [Fol16, 7.44].

A semisimple analytic group is type I by [HC54] and Ber74] in the Archimedean and non-Archimedean cases, respectively. The property of being type $\mathrm{I}$ is preserved under taking a direct product of finitely many factors [Fol16, 7.25].

Theorem 10.2 (Sauvageot density principle). Let $\nu_{n}$ be a sequence of measures on the unitary dual $\widehat{G}$. If $\nu_{n}(\widehat{\varphi}) \rightarrow \nu^{G}(\widehat{\varphi})$ for every $\varphi \in C_{c}^{\infty}(G)$ then $\nu_{n}(E) \rightarrow \nu^{G}(E)$ for every relatively quasi-compact $\nu^{G}$-regular subset $E \subset \widehat{G}$.

Proof. See Theorem 7.3 and Corollary 7.4 of Sauvageot's work Sau97. The subset $E$ is assumed to be open in [Sau97, however Shin observes Shi12] that this assumption can be removed.

10.2. Uniform lattices and relative Plancherel measure. Let $\Gamma$ be a uniform lattice in $G$. Consider a smooth compactly supported function $\varphi \in C_{c}^{\infty}(G)$ so that $\rho_{\Gamma}(\varphi)$ is a compact operator on the Hilbert space $L^{2}\left(\Gamma \backslash G, \mu_{G}\right)$ GGPS69, §1.2.2]. Moreover

$$
\operatorname{trace} \rho_{\Gamma}(\varphi)=\sum_{\pi \in \widehat{G}} m(\pi, \Gamma) \operatorname{trace} \pi(\varphi)=\operatorname{vol}(\Gamma \backslash G) \nu_{\Gamma}(\widehat{\varphi}),
$$

where $\nu_{\Gamma}$ is the relative Plancherel measure of $G$ with respect to the lattice $\Gamma$, as introduced in Definition 1.2 above.

Let $\operatorname{Sub}_{d}(G)$ denote the Chabauty subset of all the discrete subgroups in $G$. Given a function $\varphi \in C_{c}^{\infty}(G)$ we define a map $\Theta_{\varphi}$

$$
\Theta_{\varphi}: \operatorname{Sub}_{d}(G) \rightarrow \mathbb{R}, \quad \Theta_{\varphi}(\Lambda)=\sum_{\lambda \in \Lambda} \varphi(\lambda)
$$

for every discrete subgroup $\Lambda$ in $G$ discrete. In particular

$$
\Theta_{\varphi}(\{e\})=\varphi(e) \text {. }
$$

Given an identity neighborhood $U \subset G$ let $\operatorname{Sub}_{d, U}(G)$ denote the subset of $\operatorname{Sub}_{d}(G)$ consisting of those subgroups $\Delta$ satisfying $\Delta \cap U=\{e\}$.

\footnotetext{
${ }^{15}$ A function $\varphi$ on the semisimple analytic group $G$ is $s m o o t h$ if it locally a product of a $C^{\infty}$ function and a constant function on the Archimedean and non-Archimedean factors, respectively.
} 
Proposition 10.3. For every $\varphi \in C_{c}^{\infty}(G)$ the map $\Theta_{\varphi}$ is continuous on $\operatorname{Sub}_{d, U}(G)$ and admits a continuous extension to all of $\operatorname{Sub}(G)$.

Proof. Write $G=C \times D$ where $C$ and $D$ are the Archimedean and zero characteristic non-Archimedean semisimple analytic factors of $G$, respectively. $D$ admits a compact open subgroup $V_{D}$ such that every discrete subgroup in $D$ interests $V_{D}$ trivially [Ser09, LG 4.27, Th. 5] and $C$ admits an relatively compact identity neighborhood $V_{C}$ without small closed subgroups and so that $V_{C}^{2} \times V_{D} \subset U$. Denote $V=V_{C} \times V_{D}$.

Fix a compactly supported smooth function $\varphi \in C_{c}^{\infty}(G)$ and denote $Q=\operatorname{supp}(\varphi)$ so that $Q$ is compact. Cover $Q$ by finitely many translates $V_{1}, \ldots, V_{n}$ of $V$ for some $n \in \mathbb{N}$, with $V_{1}=V$. Note that every closed subgroup belonging to $\operatorname{Sub}_{d, U}(G)$ intersects each translate $V_{i}$ in at most one point.

We first show that $\Theta_{\varphi}$ is continuous on $\operatorname{Sub}_{d, U}(G)$. Let $\Delta$ be any subgroup of $G$ with $\Delta \cap U=\{e\}$. Assume without loss of generality that $\Delta \cap V_{i}=\left\{v_{i}\right\}$ for all $1 \leq i \leq m$ with some $1 \leq m \leq n$ and $\Delta \cap V_{i}=\emptyset$ for all $m<i \leq n$. Up to shrinking the neighborhood $V$ if necessary, we may moreover assume that in fact $\Delta \cap \bar{V}_{i}=\emptyset$ for all $m<i \leq n$. Taking $W$ to be an sufficiently small identity neighborhood in $G$, the value of $\Theta_{\varphi}$ on the Chabauty-open neighborhood

$$
\Omega=\bigcap_{i=1}^{m} \mathcal{O}_{2}\left(W v_{i}\right) \cap \bigcap_{i>m}^{n} \mathcal{O}_{1}\left(\bar{V}_{i}\right)
$$

of $\Delta$ can be made arbitrary close to $\Theta_{\varphi}(\Delta)$. So that $\Theta_{\varphi}$ is continuous on $\operatorname{Sub}_{d, U}$ and is moreover bounded in absolute value by $n\|\varphi\|_{\infty}$.

Note that $\operatorname{Sub}_{d, U}(G)$ is a Chabauty-closed subset of $\operatorname{Sub}(G)$, as its complement is nothing but the Chabauty-open subset $\mathcal{O}_{2}(U \backslash\{e\})$. Therefore $\Theta_{\varphi}$ can be extended to a continuous function all of $\operatorname{Sub}(G)$ by Tietze's extension theorem.

Proposition 10.4. Let $\Gamma$ be a uniform lattice in $G$. Let $\mu_{\Gamma}$ be the invariant random subgroup and $\nu_{\Gamma}$ the relative Plancherel measure on $\widehat{G}$ associated to $\Gamma$. Then $\nu_{\Gamma}(\widehat{\varphi})=\mu_{\Gamma}\left(\Theta_{\varphi}\right)$ for every function $\varphi \in C_{c}^{\infty}(G)$.

Proof. In view of Proposition 10.3 we may extend $\Theta_{\varphi}$ to a Chabauty-continuous function on $\operatorname{Sub}(G)$. The equality $\nu_{\Gamma}(\widehat{\varphi})=\mu_{\Gamma}\left(\Theta_{\varphi}\right)$ follows from the computation on p. 37-38 of $\left[\mathrm{ABB}^{+} 17\right]$. This argument of $\left[\mathrm{ABB}^{+} 17\right]$ is general and applies to uniform lattices in any l.c.s.c. group.

10.3. Proof of convergence for relative Plancherel measures. We conclude this section by providing proofs of Theorem 1.3 and Corollary 1.4 .

Let $G$ be a semisimple analytic group in zero characteristic. Let $\Gamma_{n}$ be a uniformly discrete sequence of lattices in $G$ so that the corresponding invariant random subgroups $\mu_{\Gamma_{n}}$ converge to $\delta_{\{e\}}$. Let $\nu^{G}$ and $\nu_{n}$ denote the Plancherel measure of $G$ and the relative normalized Placherel measures associated to the $\Gamma_{n}$ 's, respectively.

Proof of Theorem 1.3. In view of the Sauvageot density principle to prove Theorem 1.3 we need to show that $\nu_{n}(\widehat{\varphi}) \rightarrow \nu^{G}(\widehat{\varphi})$ for every function $\varphi \in C_{c}^{\infty}(G)$. Consider a fixed function $\varphi \in C_{c}^{\infty}(G)$ and regard $\Theta_{\varphi}$ as a continuous function on $\operatorname{Sub}(G)$ making use of Proposition 10.3 In light of the Plancherel formula, Proposition 10.4, and the weak-* convergence of $\mu_{\Gamma_{n}}$ to $\delta_{\{e\}}$ we obtain that

$$
\nu_{n}(\widehat{\varphi})=\mu_{n}\left(\Theta_{\varphi}\right) \stackrel{n \rightarrow \infty}{\longrightarrow} \delta_{\{e\}}\left(\Theta_{\varphi}\right)=\Theta_{\varphi}(\{e\})=\varphi(e)=\nu^{G}(\widehat{\varphi})
$$


as required.

Proof of Corollary 1.4. To deduce the corollary from Theorem 1.3 we need to show that every singleton $\{\pi\} \subset \widehat{G}$ is relatively compact and $\nu^{G}$-regular.

The group $G$ is limina $\sqrt{16}$ in the sense that $\pi(\varphi)$ is a compact operator for every function $\varphi \in C_{c}^{\infty}(G)$. It is shown in Gli61] that the dual space $\widehat{G}$ of a liminal group is $T_{1}$. In particular every singleton $\{\pi\} \subset \widehat{G}$ is closed and so relatively compact.

Since $G$ is l.c.s.c. it follows from the definitions that Fell's topology on $\widehat{G}$ is first countable. Recall that in a $T_{1}$-space every subset is equal to the intersection of the open sets containing it. Therefore the singleton $\{\pi\}$ is equal to the intersection of a countable family of open sets. Moreover Fell's topology is locally compact and compact subsets have finite Plancherel measure [Dix77, 18.1.2, 18.8.4]. Therefore $\{\pi\}$ is $\nu^{G}$-regular, as required.

\section{REFERENCES}

[AB94] Norbert A'Campo and Marc Burger. Réseaux arithmétiques et commensurateur d'après G.A. Margulis. Inventiones mathematicae, 116(1):1-25, 1994.

$\left[\mathrm{ABB}^{+} 17\right]$ Miklos Abert, Nicolas Bergeron, Ian Biringer, Tsachik Gelander, Nikolay Nikolov, Jean Raimbault, and Iddo Samet. On the growth of $l^{2}$-invariants for sequences of lattices in Lie groups. Annals of Mathematics, 185(3):711-790, 2017.

[Ber74] Joseph Bernstein. All reductive $p$-adic groups are tame. Funktsional'nyi Analiz $i$ ego Prilozheniya, 8(2):3-6, 1974.

[BG14] Uri Bader and Tsachik Gelander. Equicontinuous actions of semisimple groups. To appear in Groups Geometry and Dynamics., 2014.

[BH11] Martin R Bridson and André Haefliger. Metric spaces of non-positive curvature, volume 319. Springer Science \& Business Media, 2011.

[BL04] Y Barnea and M Larsen. Random generation in semisimple algebraic groups over local fields. Journal of Algebra, 271(1):1-10, 2004.

[Bor12] Armand Borel. Linear algebraic groups, volume 126. Springer Science \& Business Media, 2012.

[BP89] Armand Borel and Gopal Prasad. Finiteness theorems for discrete subgroups of bounded covolume in semi-simple groups. Publications Mathématiques de l'IHÉS, 69:119-171, 1989.

[Bra45] Jean Braconnier. Sur les groupes topologiques localement compacts. 1945.

[BT73] Armand Borel and Jacques Tits. Homomorphismes "abstraits" de groupes algebriques simples. Annals of Mathematics, pages 499-571, 1973.

[CC15] Pierre-Emmanuel Caprace and Corina Ciobotaru. Gelfand pairs and strong transitivity for Euclidean buildings. Ergodic Theory and Dynamical Systems, 35(04):10561078, 2015.

[CEG86] Richard Douglas Canary, David BA Epstein, and P Gree. Notes on notes of Thurston. University of Warwick, 1986.

[DDSMS03] John D Dixon, Marcus PF Du Sautoy, Avinoam Mann, and Dan Segal. Analytic pro-p groups, volume 61. Cambridge University Press, 2003.

[DGLL15] Bruno Duchesne, Yair Glasner, Nir Lazarovich, and Jean Lécureux. Geometric density for invariant random subgroups of groups acting on CAT(0) spaces. Geometriae Dedicata, 175(1):249-256, 2015.

[Dix77] Jacques Dixmier. $C *$-algebras. translated from the French by Francis Jellett. NorthHolland Math. Library, 15, 1977.

[Ele10] Gábor Elek. Betti numbers are testable. In Fete of Combinatorics and Computer Science, pages 139-149. Springer, 2010.

[Fol16] Gerald B Folland. A course in abstract harmonic analysis, volume 29. CRC press, 2016 .

${ }^{16}$ A l.c.s.c. group $G$ is type $\mathrm{I}$ or is liminal if and only if $\widehat{G}$ is $T_{0}$ or $T_{1}$ in the Fell topology, respectively. In particular being liminal is a stronger property than type I. 
[Fur76] Harry Furstenberg. A note on Borels density theorem. Proceedings of the American Mathematical Society, 55(1):209-212, 1976.

[Gar97] Paul B Garrett. Buildings and classical groups. CRC Press, 1997.

[Gel15] Tsachik Gelander. Kazhdan-Margulis theorem for invarant random subgroups. To appear in Advances in Mathematics, 2015.

[GGPS69] I Gelfand, M Graev, and I Piatetski-Shapiro. Representation theory and automorphic forms. WB Saunders Co., Philadelphia, 1969.

[GL16] Tsachik Gelander and Arie Levit. Local rigidity of uniform lattices. arXiv preprint arXiv:1605.01693, 2016

[Gli61] James Glimm. Type I $C^{*}$-algebras. Annals of mathematics, pages 572-612, 1961.

[GM11] Tsachik Gelander and Yair Minsky. The dynamics of $\operatorname{Aut}\left(F_{n}\right)$ on redundant representations. arXiv preprint arXiv:1104.4774, 2011.

[Gol09] Alireza Salehi Golsefidy. Lattices of minimum covolume in Chevalley groups over local fields of positive characteristic. Duke Mathematical Journal, 146(2):227-251, 2009.

[GS10] Paul Gartside and Michael Smith. Counting the closed subgroups of profinite groups. Journal of Group Theory, 13(1):41-61, 2010.

[GW97] E Glasner and B Weiss. Kazhdan's property T and the geometry of the collection of invariant measures. Geometric \& Functional Analysis GAFA, 7(5):917-935, 1997.

[HC54] Harish-Chandra. Representations of semisimple Lie groups. III. Trans. Amer. Math. Soc., 76:234253, 1954.

[KM68] David Kazhdan and Gregory Margulis. A proof of Selbergs hypothesis. Mat. Sb.(NS), 75(117):163-168, 1968.

[Lev17] Arie Levit. The Nevo-Zimmer intermediate factor theorem over local fields. Geometriae Dedicata, 186(1):149-171, February 2017.

[Lüc94] Wolfgang Lück. Approximating $L^{2}$-invariants by their finite-dimensional analogues. Geometric \& Functional Analysis GAFA, 4(4):455-481, 1994.

[Mar77] Gregory A Margulis. Cobounded subgroups of algebraic groups over local fields. Functional Analysis and Its Applications, 11(2):119-128, 1977.

[Mar91] Gregori A Margulis. Discrete subgroups of semisimple Lie groups, volume 17. Springer Science \& Business Media, 1991.

[Pet13] Henrik Densing Petersen. L2-betti numbers of locally compact groups. Comptes Rendus Mathematique, 351(9):339-342, 2013.

[Pin98] Richard Pink. Compact subgroups of linear algebraic groups. Journal of Algebra, 206(2):438-504, 1998.

[Pin04] Richard Pink. On Weil restriction of reductive groups and a theorem of Prasad. Mathematische Zeitschrift, 248(3):449-457, 2004.

[PR72] Gopal Prasad and Madabusi Santanam Raghunathan. Cartan subgroups and lattices in semi-simple groups. Annals of Mathematics, pages 296-317, 1972.

[PR92] Vladimir Petrovich Platonov and Andrei Stepanovich Rapinchuk. Algebraic groups and number theory. Russian Mathematical Surveys, 47(2):133-161, 1992.

[Pra82] Gopal Prasad. Elementary proof of a theorem of Bruhat-Tits-Rousseau and of a theorem of Tits. Bulletin de la Société mathématique de France, 110:197-202, 1982.

[PST16] Henrik Densing Petersen, Roman Sauer, and Andreas Thom. $l^{2}$-betti numbers of totally disconnected groups and their approximation by betti numbers of lattices. arXiv preprint arXiv:1612.04559, 2016.

[Rag76] M-S Raghunathan. Discrete subgroups of Lie groups. Springer-Verlag, PWN-Polish Scientific Publishers; Warsaw, 1976.

[RZ00] Luis Ribes and Pavel Zalesskii. Profinite groups. In Profinite Groups, pages 19-77. Springer, 2000.

[Sau97] François Sauvageot. Principe de densité pour les groupes rèuctifs. Compositio Mathematica, 108:151-184, 1997.

[Ser09] Jean-Pierre Serre. Lie algebras and Lie groups. Springer, 2009.

[Shi12] Sug Woo Shin. Automorphic Plancherel density theorem. Israel Journal of Mathematics, 192(1):83-120, 2012.

[SZ94] Garrett Stuck and Robert J Zimmer. Stabilizers for ergodic actions of higher rank semisimple groups. Annals of Mathematics, pages 723-747, 1994. 
[Tit66] Jacques Tits. Classification of algebraic semisimple groups. In Proc. Sympos. Pure Math, volume 9, pages 33-62, 1966.

[Wan72] Hsien Chung Wang. Topics on totally discontinuous groups. Symmetric spaces (Short Courses, Washington Univ., St. Louis, Mo., 1969-1970), 8:459-487, 1972. 